\title{
Phenomenological studies on the $B_{d, s}^{0} \rightarrow J / \psi f_{0}(500)\left[f_{0}(980)\right]$ decays
}

\author{
Xin Liu \\ Department of Physics, Jiangsu Normal University, Xuzhou 221116, China \\ Zhi-Tian Zou and Ying Li \\ Department of Physics, Yantai University, Yantai 264005, China \\ Zhen-Jun Xiao \\ Department of Physics and Institute of Theoretical Physics, Nanjing Normal University, \\ Nanjing 210023, China
}

(Received 11 June 2019; published 29 July 2019)

\begin{abstract}
Encouraged by the global agreement between theoretical predictions and experimental measurements for $B \rightarrow J / \psi V$ decays, we extend that perturbative QCD formalism to $B_{d, s}^{0} \rightarrow J / \psi f_{0}(500)\left[f_{0}(980)\right]$ decays at the presently known next-to-leading order in the quark-antiquark description of $f_{0}(500)$ and $f_{0}(980)$. With the angle $\phi_{f} \approx 25^{\circ}$ of the $f_{0}(500)-f_{0}(980)$ mixing in the quark-flavor basis, we find that the branching ratios of the $B_{d}^{0} \rightarrow J / \psi f_{0}(500)\left(\rightarrow \pi^{+} \pi^{-}\right)$and $B_{d, s}^{0} \rightarrow J / \psi f_{0}(980)\left(\rightarrow \pi^{+} \pi^{-}\right)$modes generally agree with the current data or the upper limits within uncertainties, except for the seemingly challenging $B_{s}^{0} \rightarrow$ $J / \psi f_{0}(500)\left(\rightarrow \pi^{+} \pi^{-}\right)$one. Then, we further explore the relevant observables of the $B_{d, s}^{0} \rightarrow$ $J / \psi f_{0}(500)\left[f_{0}(980)\right]$ decays, which could provide further constraints on the mixing angle $\phi_{f}$ and/or $\mathrm{SU}(3)$ flavor symmetry breaking effects. As a byproduct, we predict $\mathrm{BR}\left(B_{d}^{0} \rightarrow J / \psi f_{0}(980)\left(\rightarrow K^{+} K^{-}\right)\right)=$ $5.8_{-2.9}^{+3.1} \times 10^{-7}$ and $\operatorname{BR}\left(B_{s}^{0} \rightarrow J / \psi f_{0}(980)\left(\rightarrow K^{+} K^{-}\right)\right)=4.6_{-2.3}^{+2.6} \times 10^{-5}$. All theoretical predictions await the future examinations with high precision.
\end{abstract}

DOI: 10.1103/PhysRevD.100.013006

\section{INTRODUCTION}

It is well known that the golden modes $B_{d}^{0} \rightarrow J / \psi K_{S}$ and $B_{s}^{0} \rightarrow J / \psi \phi$ in the heavy $b$ flavor sector provide an ideal ground to test the standard model(SM) and search for the possible new physics beyond SM. Because of the expected small penguin pollution, the above two decays can usually offer good opportunities to extract the weak phases $\phi_{d}$ and $\phi_{s}$ [or the Cabibbo-Kobayashi-Maskawa (CKM) angles $\beta_{d}$ and $\beta_{s}$ ] from the indirect $C P$-violating asymmetries in the neutral $B_{d}^{0}-\bar{B}_{d}^{0}$ and $B_{s}^{0}-\bar{B}_{s}^{0}$ mixings, respectively. Note that the significant nonzero deviations experimentally to the SM predictions for the interesting $\sin \phi_{d}$ and $\sin \phi_{s}$ would indicate the exotic new physics beyond SM, and especially the latter one is of great interest. However, it is stressed that the $B_{s}^{0} \rightarrow J / \psi \phi$ final state contains two vector mesons, which lead to a mixture of $C P$-even and $C P$-odd eigenstates; then a complicated angular decomposition is

Published by the American Physical Society under the terms of the Creative Commons Attribution 4.0 International license. Further distribution of this work must maintain attribution to the author(s) and the published article's title, journal citation, and DOI. Funded by SCOAP ${ }^{3}$. required to analyze the relevant observables. Consequently, the extraction of the $B_{s}^{0}-\bar{B}_{s}^{0}$ mixing phase $\phi_{s}$ suffers from large errors. Therefore, some new alternative channels are proposed and, in particular, the $B_{s}^{0} \rightarrow J / \psi f_{0}(980)$ [For simplicity, $f_{0}(980)$ is abbreviated as $f_{0}$ in the following context unless otherwise stated.] is believed to have the supplementary power to significantly reduce the error of $\phi_{s}$ [1-3]. The underlying reason is that $f_{0}$ is a $0^{++}$scalar state [for example, see the minireview on scalar mesons coming from the Particle Data Group (PDG) in [4]], and thus the final state $J / \psi f_{0}$ is a $C P$ eigenstate, which means that, relative to the $B_{s}^{0} \rightarrow J / \psi \phi$ channel, there are no needs to perform an angular analysis, and therefore the relevant analysis is simplified greatly. Indeed, this point has been proven in the relevant measurements, for example, the latest one in Ref. [5].

Presently, this alternative channel $B_{s}^{0} \rightarrow J / \psi f_{0}$ has been searched through the resonant contribution with $f_{0} \rightarrow$ $\pi^{+} \pi^{-}$by a variety of groups experimentally. Meanwhile, the expected mixing partner $f_{0}(500)$, like $\eta-\eta^{\prime}$ mixing in the pseudoscalar sector, was examined in the $B_{d}^{0} \rightarrow$ $J / \psi f_{0}(500)$ decay [hereafter, $f_{0}(500)$ is denoted as $\sigma$ for convenience] by the Large Hadron Collider beauty (LHCb) Collaboration also through resonance studies [6,7]. 
The available measurements of branching ratios for the considered $B_{d}^{0} \rightarrow J / \psi \sigma$ and $B_{s}^{0} \rightarrow J / \psi f_{0}$ decays are as follows [4,7-9],

$$
\mathrm{BR}\left(B_{d}^{0} \rightarrow J / \psi f_{0}(500), f_{0} \rightarrow \pi^{+} \pi^{-}\right)=8.8_{-1.6}^{+1.2} \times 10^{-6},
$$

$$
\mathrm{BR}\left(B_{s}^{0} \rightarrow J / \psi f_{0}(980), f_{0} \rightarrow \pi^{+} \pi^{-}\right)=1.28_{-0.18}^{+0.18} \times 10^{-4}
$$

The precision of relevant measurements will be rapidly improved along with more and more data samples collected at the LHCb and/or Belle-II experiments in the near future. Moreover, the upper limits for $\operatorname{BR}\left(B_{d}^{0} \rightarrow J / \psi f_{0}\right)$ and $\operatorname{BR}\left(B_{s}^{0} \rightarrow J / \psi \sigma\right)$ are also made currently by the LHCb Collaboration as follows $[6,10]$ :

$$
\begin{gathered}
\mathrm{BR}\left(B_{d}^{0} \rightarrow J / \psi f_{0}(980), f_{0} \rightarrow \pi^{+} \pi^{-}\right) \\
=6.1_{-2.4}^{+3.5} \times 10^{-7}<1.1 \times 10^{-6}, \\
\mathrm{BR}\left(B_{s}^{0} \rightarrow J / \psi f_{0}(500), f_{0} \rightarrow \pi^{+} \pi^{-}\right)<4 \times 10^{-6} .
\end{gathered}
$$

It is necessary to stress that the $\mathrm{LHCb}$ results for $B_{s}^{0}$ decays correspond to the time-integrated quantities, while theory predictions refer to the branching fractions at $t=0$ [11], and may differ by $10 \%$.

Furthermore, an interesting ratio $R_{f_{0} / \phi}$ between the branching ratios of the alternative $B_{s}^{0} \rightarrow J / \psi f_{0}$ and the golden $B_{s}^{0} \rightarrow J / \psi \phi$ channels is defined as [1]

$$
R_{f_{0} / \phi} \equiv \frac{\mathrm{BR}\left(B_{s}^{0} \rightarrow J / \psi f_{0}, f_{0} \rightarrow \pi^{+} \pi^{-}\right)}{\operatorname{BR}\left(B_{s}^{0} \rightarrow J / \psi \phi, \phi \rightarrow K^{+} K^{-}\right)},
$$

which has been measured by various groups and the related results are collected as the following [9,12-15],

$$
\begin{aligned}
R_{f_{0} / \phi} \equiv & \frac{\operatorname{BR}\left(B_{s}^{0} \rightarrow J / \psi f_{0}, f_{0} \rightarrow \pi^{+} \pi^{-}\right)}{\operatorname{BR}\left(B_{s}^{0} \rightarrow J / \psi \phi, \phi \rightarrow K^{+} K^{-}\right)} \\
= & \begin{cases}0.252_{-0.046}^{+0.053} & (\mathrm{LHCb}), \\
0.257_{-0.024}^{+0.024} & (\mathrm{CDF}), \\
0.275_{-0.073}^{+0.073} & (\mathrm{D} 0), \\
0.140_{-0.024}^{+0.024} & (\mathrm{CMS}), \\
0.207_{-0.016}^{+0.016} & \text { (HFLAV }) .\end{cases}
\end{aligned}
$$

Meanwhile, another ratio between $\operatorname{BR}\left(B_{s}^{0} \rightarrow J / \psi f_{0}, f_{0} \rightarrow\right.$ $\left.\pi^{+} \pi^{-}\right)$and $\operatorname{BR}\left(B_{s}^{0} \rightarrow J / \psi \phi\right)$ from different groups is read as follows $[4,9,13,14,16]$,

$$
\begin{aligned}
& \frac{\operatorname{BR}\left(B_{s}^{0} \rightarrow J / \psi f_{0}, f_{0} \rightarrow \pi^{+} \pi^{-}\right)}{\operatorname{BR}\left(B_{s}^{0} \rightarrow J / \psi \phi\right)} \\
& = \begin{cases}0.069_{-0.012}^{+0.012} & (\mathrm{CMS}), \\
0.139_{-0.013}^{+0.026} & (\mathrm{LHCb}), \\
0.135_{-0.036}^{+0.036} & \text { (D0), } \\
0.126_{-0.012}^{+0.012} & \text { (CDF), } \\
0.119_{-0.014}^{+0.013} & \text { (PDG Fit), } \\
0.111_{-0.018}^{+0.020} & \text { (PDG Average) } .\end{cases}
\end{aligned}
$$

These data would be helpful to explore the dynamics involved in the $B_{s}^{0} \rightarrow J / \psi f_{0}$ decay and to identify the inner structure or the components of the scalar $f_{0}$ state.

It is believed that light scalars below $1 \mathrm{GeV}$ could play an important role to help understand the QCD vacuum because of their same quantum numbers $J^{P C}=0^{++}$[17]. But, it is unfortunate that the inner structure of these light scalars such as $\sigma$ and $f_{0}$ is presently hard to understood well due to the complicated nonperturbative QCD dynamics. Therefore, the interpretation of their components is far from being straightforward and still in controversy; e.g., see reviews [4,18-23]. Alternatively, however, the production of $\sigma$ and $f_{0}$ in the heavy $D_{(s)}, B_{(s)}$, even $B_{c}$ meson decays could provide another insight into their inner structure. In particular, the $B_{d, s}^{0} \rightarrow J / \psi \sigma\left(f_{0}\right)$ decays could be more favored because they contain few topologies of Feynman diagrams, as well as the expectantly small penguin pollution. For example, Stone and Zhang ever suggested in Ref. [24] that these channels could be used to discern the $q \bar{q}$ or tetraquark nature of scalars, and an upper limit of the mixing angle between $\sigma$ and $f_{0}$ was provided with the help of $B_{d}^{0} \rightarrow J / \psi \sigma$ and $B_{s}^{0} \rightarrow J / \psi f_{0}$ decays as $29^{\circ}$ at $90 \%$ confidence level for the $\sigma$ and $f_{0}$ being $q \bar{q}$ states.

On the theoretical side, some of these $B_{d, s}^{0} \rightarrow J / \psi \sigma\left(f_{0}\right)$ modes have been investigated to a different extent with different methods/approaches in the literature [17,25-35], and, in particular,

(a) Colangelo et al. studied the $B_{s}^{0} \rightarrow J / \psi f_{0}$ decay by using the light-cone QCD sum rule and factorization assumption in Ref. [25] with leading order prediction $\operatorname{BR}\left(B_{s}^{0} \rightarrow J / \psi f_{0}\right)=3.1 \pm 2.4 \times 10^{-4}$ and the nextto-leading order (NLO) one $\operatorname{BR}\left(B_{s}^{0} \rightarrow J / \psi f_{0}\right)=$ $5.3 \pm 3.9 \times 10^{-4}$, and using generalized factorization and SU(3) flavor symmetry in Ref. [26] with different branching ratios $4.7 \pm 1.9 \times 10^{-4}$ and $2.0 \pm 0.8 \times 10^{-4}$, respectively. Notice that here $f_{0}$ was assumed as a pure $s \bar{s}$ state.

(b) By assuming $f_{0}$ as an $s \bar{s}$ state, Leitner et al. estimated the $B_{s}^{0} \rightarrow J / \psi f_{0}$ decay rate around $5.0 \times 10^{-4}$ in the QCD factorization approach [27], based on reproduction of the data about $\operatorname{BR}\left(B_{s}^{0} \rightarrow J / \psi \phi\right)$.

(c) Fleischer et al. showed the anatomy of $B_{d, s}^{0} \rightarrow$ $J / \psi f_{0}$ in Ref. [28] by considering the $q \bar{q}$ and 
tetraquark pictures of the $f_{0}$ state. And they obtained the branching ratios with different mixing angles $\varphi_{M}$ in the conventional two-quark picture: $\left.\mathrm{BR}\left(B_{s}^{0} \rightarrow J / \psi f_{0}\right)\right|_{\varphi_{M}=0^{\circ}} \simeq 1.9 \times 10^{-4}$ and $\operatorname{BR}\left(B_{s}^{0} \rightarrow\right.$ $\left.J / \psi f_{0}\right)\left.\right|_{\varphi_{M}=41.6^{\circ}} \simeq 4.8 \times 10^{-4}$ by using factorization approximation and $\mathrm{SU}(3)$ flavor symmetry. Meanwhile, the $B_{d}^{0} \rightarrow J / \psi f_{0}\left(\rightarrow \pi^{+} \pi^{-}\right)$decay rate $\sim 1.65_{-0.29}^{+0.34} \times 10^{-6}$ was also predicted.

(d) Under the assumption of two-quark structure and the $\sigma-f_{0}$ mixing, Li et al. studied the $B_{s}^{0} \rightarrow$ $J / \psi \sigma\left(f_{0}\right)$ decays with a mixed "QCD factorization plus perturbative QCD (PQCD) factorization” approach [29] and predicted the branching ratios $\mathrm{BR}\left(B_{s}^{0} \rightarrow J / \psi f_{0}\right)=2.43_{-0.31}^{+0.30} \times 10^{-4}$ and $\mathrm{BR}\left(B_{s}^{0} \rightarrow\right.$ $J / \psi \sigma)=4.72_{-0.59}^{+0.62} \times 10^{-5}$, corresponding to the mixing angle $\phi_{f}$ about $\pm 34^{\circ}$.

In light of the current measurements on various observables performed by the LHCb Collaboration with good precision, it is essential to make a systematic investigation on all of the $B_{d, s}^{0} \rightarrow J / \psi \sigma\left(f_{0}\right)$ modes. Encouraged by the global agreement between the data and the theoretical predictions in the PQCD approach [36-39] on the $B \rightarrow$ $J / \psi V$ decays at the NLO accuracy [40], we extend that formalism to the $B_{d, s}^{0} \rightarrow J / \psi \sigma\left(f_{0}\right)$ decays in the quarkantiquark description of $\sigma$ and $f_{0}$ with including the known NLO corrections in $\alpha_{s}$, namely, the vertex corrections. It is well known that, as one of the popular factorization methods based on QCD dynamics, the PQCD approach has been widely employed to calculate the hadronic matrix elements in the nonleptonic decays of heavy $b$ quark mesons. Because of the introduction of the Sudakov factors arising from $k_{T}$ resummation [41,42] and threshold resummation $[43,44]$, respectively, the PQCD approach could be utilized to compute the nonfactorizable emission and the annihilation diagrams safely, apart from the factorizable emission ones. With the perturbative calculations of both tree and penguin amplitudes in the PQCD approach, we could provide the predictions on the observables such as the $C P$-averaged branching ratios, the $C P$-violating asymmetries, and so forth with much more reliability. Hence, these reliable calculations would help us to further investigate the

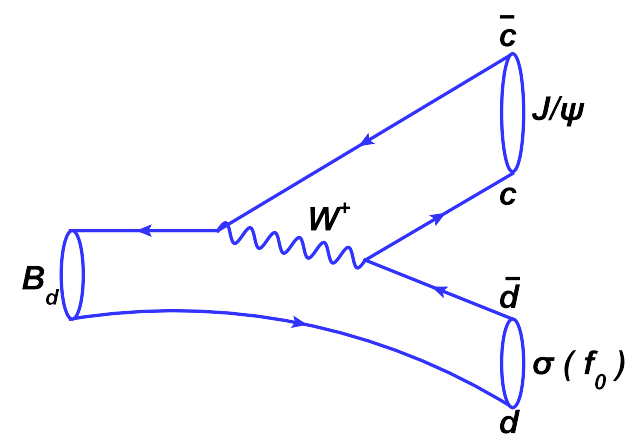

impact of the penguin contributions to the $C P$ asymmetry measurements, even the extraction of weak phases $\phi_{d, s}$, and explore the useful information such as the mixing angle $\phi_{f}$ between the mixtures of $\sigma$ and $f_{0}$, if they are really the $q \bar{q}$ mesons.

The rest of this paper is organized as follows: After this introduction, Sec. II is devoted to the analysis of decay amplitudes for the $B_{d, s}^{0} \rightarrow J / \psi \sigma\left(f_{0}\right)$ modes in the PQCD approach. The essential nonperturbative inputs are also collected in this section. The numerical results and phenomenological analyses for the $C P$-averaged branching ratios, $C P$-violating asymmetries, and other interesting observables of the considered decays are given in Sec. III. As a byproduct, we also present the $C P$-averaged branching ratios of $B_{d, s}^{0} \rightarrow J / \psi f_{0}\left(\rightarrow K^{+} K^{-}\right)$decays in this section. We summarize this work and conclude in Sec. IV.

\section{DECAY AMPLITUDES OF $B_{d, s}^{0} \rightarrow J / \psi \sigma\left(f_{0}\right)$ AND ESSENTIAL INPUTS}

Similar to $B_{d, s}^{0} \rightarrow J / \psi \eta\left(\eta^{\prime}\right)$ decays in the pseudoscalar sector [45], the leading quark-level Feynman diagrams contributing to the $B_{d, s}^{0} \rightarrow J / \psi \sigma\left(f_{0}\right)$ decays have been illustrated in Fig. 1. Before writing down the decay amplitudes of the considered $B_{d, s}^{0} \rightarrow J / \psi \sigma\left(f_{0}\right)$ channels, it is essential to make some remarks on the mixing between $\sigma$ and $f_{0}$. Analogous to the $\eta-\eta^{\prime}$ mixing, this scalar $\sigma-f_{0}$ mixing can also be described by a $2 \times 2$ rotation matrix with a single angle $\phi_{f}$ in the quark-flavor basis, namely,

$$
\left(\begin{array}{c}
\sigma \\
f_{0}
\end{array}\right)=\left(\begin{array}{cc}
\cos \phi_{f} & -\sin \phi_{f} \\
\sin \phi_{f} & \cos \phi_{f}
\end{array}\right)\left(\begin{array}{c}
f_{q} \\
f_{s}
\end{array}\right),
$$

with the quark-flavor states $f_{q} \equiv \frac{u \bar{u}+d \bar{d}}{\sqrt{2}}$ and $f_{s} \equiv s \bar{s}$. Various mixing angle $\phi_{f}$ measurements have been derived and summarized in the literature with a wide range of values; for example, see Refs. [28,46-48]. However, it is worth of pointing out that, based on the recent measurement and the accompanied discussion performed by the LHCb Collaboration [6], the upper limits $\left|\phi_{f}\right|<31^{\circ}$ have been set for the first time in the $B$ meson decays with a

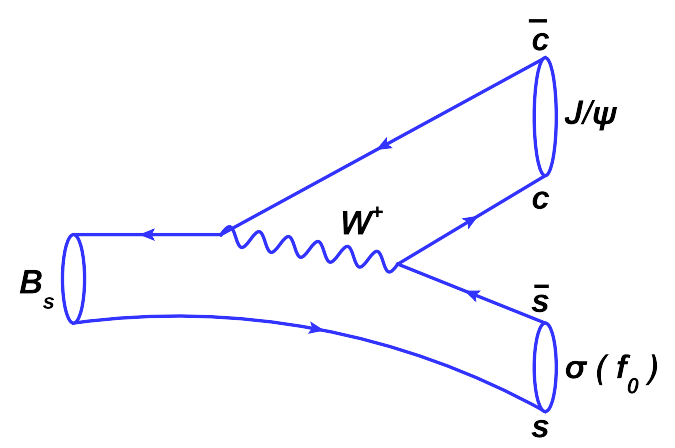

FIG. 1. Leading quark-level Feynman diagrams contributing to the $B_{d, s}^{0} \rightarrow J / \psi \sigma\left(f_{0}\right)$ decays. 
two-quark structure description of $\sigma$ and $f_{0}$. Therefore, in other words, the agreement of $C P$-averaged branching ratios for the $B_{d, s}^{0} \rightarrow J / \psi \sigma\left(f_{0}\right)$ decays between the experimental measurements and the PQCD predictions in this work is expected to provide some useful information to further constrain the possible range of this $\phi_{f}$ angle.

According to the aforementioned mixing pattern, the $B_{d, s}^{0} \rightarrow J / \psi \sigma\left(f_{0}\right)$ decay amplitudes could then be written explicitly with the help of $B_{d(s)}^{0} \rightarrow J / \psi f_{q(s)}$ as follows,

$$
\begin{gathered}
\mathcal{A}\left(B_{d}^{0} \rightarrow J / \psi \sigma\right)=\mathcal{A}\left(B_{d}^{0} \rightarrow J / \psi f_{q}\right) \cdot \cos \phi_{f}, \\
\mathcal{A}\left(B_{d}^{0} \rightarrow J / \psi f_{0}\right)=\mathcal{A}\left(B_{d}^{0} \rightarrow J / \psi f_{q}\right) \cdot \sin \phi_{f} ; \\
\mathcal{A}\left(B_{s}^{0} \rightarrow J / \psi \sigma\right)=\mathcal{A}\left(B_{s}^{0} \rightarrow J / \psi f_{s}\right) \cdot\left(-\sin \phi_{f}\right), \\
\mathcal{A}\left(B_{s}^{0} \rightarrow J / \psi f_{0}\right)=\mathcal{A}\left(B_{s}^{0} \rightarrow J / \psi f_{s}\right) \cdot \cos \phi_{f},
\end{gathered}
$$

which yield the following relations:

$$
\begin{aligned}
& \left|\mathcal{A}\left(B_{d}^{0} \rightarrow J / \psi \sigma\right)\right|^{2}+\left|\mathcal{A}\left(B_{d}^{0} \rightarrow J / \psi f_{0}\right)\right|^{2} \\
& \quad=\left|\mathcal{A}\left(B_{d}^{0} \rightarrow J / \psi f_{q}\right)\right|^{2}, \\
& \left|\mathcal{A}\left(B_{s}^{0} \rightarrow J / \psi \sigma\right)\right|^{2}+\left|\mathcal{A}\left(B_{s}^{0} \rightarrow J / \psi f_{0}\right)\right|^{2} \\
& \quad=\left|\mathcal{A}\left(B_{s}^{0} \rightarrow J / \psi f_{s}\right)\right|^{2} .
\end{aligned}
$$

Here, the decay amplitudes of $B_{d(s)}^{0}$ decaying into the flavor state $f_{q(s)}$ could be easily obtained from those in the $B_{d(s)}^{0} \rightarrow J / \psi \omega(\phi)$ modes correspondingly in the PQCD approach, which is clarified later. These formulas indicate that the theoretically reliable estimates of the perturbative and nonperturbative QCD dynamics in the $B_{d(s)}^{0} \rightarrow$ $J / \psi f_{q(s)}$ modes are very important to understand the $B_{d, s}^{0} \rightarrow J / \psi \sigma\left(f_{0}\right)$ decays experimentally, and vice versa. It is worth mentioning that the wave functions associated with light-cone distribution amplitudes that describe the hadronization of valence quark and valence antiquark in a meson are the only nonperturbative inputs in the PQCD calculations and are processes independent. It is fortunate that the nonperturbative QCD dynamics of the abovementioned initial and final hadrons has been investigated in the literature.

(a) It is remarked that the $B \rightarrow J / \psi P(V)$ decays $[P(V)$ stands for the light pseudoscalar (vector) mesons] have been studied in the PQCD approach at the NLO accuracy $[40,45,49-52]$ with the same wave functions and distribution amplitudes for the heavy $B_{d, s}^{0}$ and $J / \psi$ mesons. Furthermore, the general consistency between theory and experiment in the SM for the branching ratios of those considered decays has been obtained. Thus, in this work, we adopt the same wave functions and distribution amplitudes of $B_{d, s}^{0}$ and $J / \psi$ as those used in, for example, Ref. [40] and references therein, as well as the relevant hadronic parameters.

(b) For the scalar flavor states $f_{q}$ and $f_{s}$, the light-cone wave function can generally be defined as [53]

$$
\begin{aligned}
\Phi_{f_{q(s)}}(x)= & \frac{i}{\sqrt{2 N_{c}}}\left\{P / \phi_{f_{q(s)}}(x)+m_{f_{q(s)}} \phi_{f_{q(s)}^{S}}^{S}(x)\right. \\
& \left.+m_{f_{q(s)}}(n / v /-1) \phi_{f_{q(s)}^{T}}^{T}(x)\right\}_{\alpha \beta},
\end{aligned}
$$

where $N_{c}, \phi_{f_{q(s)}}$, and $\phi_{f_{q(s)}}^{S, T}, m_{f_{q(s)}}, n$, and $v$, and $\alpha, \beta$ are the color factor, the leading twist, and twist-3 distribution amplitudes, the mass of $f_{q(s)}$, the dimensionless lightlike unit vectors $n=\left(1,0, \mathbf{0}_{T}\right)$ and $v=\left(0,1, \mathbf{0}_{T}\right)$, and the color indices, respectively, while $x$ denotes the momentum fraction carried by the quark in the meson.

The light-cone distribution amplitudes up to twist 3 as shown in Eq. (15) have been investigated in the QCD sum rule technique ${ }^{1}$ [53] with the contributions arising from only the odd Gegenbauer polynomials,

$$
\begin{aligned}
& \phi_{f_{q(s)}}= \frac{\bar{f}_{f_{q(s)}}(\mu)}{2 \sqrt{2 N_{c}}}\left\{6 x ( 1 - x ) \left[B_{1}^{q(s)}(\mu) C_{1}^{3 / 2}(2 x-1)\right.\right. \\
&+\left.\left.B_{3}^{q(s)}(\mu) C_{3}^{3 / 2}(2 x-1)\right]\right\} \\
& \phi_{f_{q(s)}}^{S}=\frac{1}{2 \sqrt{2 N_{c}}} \bar{f}_{f_{q(s)}}(\mu) \\
& \phi_{f_{q(s)}^{T}}^{T}=\frac{1}{2 \sqrt{2 N_{c}}} \bar{f}_{f_{q(s)}}(\mu)(1-2 x)
\end{aligned}
$$

where the scalar decay constants $\bar{f}_{f_{q}}(\mu)$ and $\bar{f}_{f_{s}}(\mu)$ and the Gegebnbauer moments $B_{1,3}^{q(s)}(\mu)$ at the normalization scale $\mu=1 \mathrm{GeV}$ are as follows [53]:

$$
\begin{aligned}
\bar{f}_{f_{q}} \simeq 0.35 \mathrm{GeV}, \quad \bar{f}_{f_{s}} \simeq 0.33 \mathrm{GeV} ; \\
B_{1}^{q}=-0.92 \pm 0.08, \quad B_{3}^{q}=-1.00 \pm 0.05 \\
B_{1,3}^{s} \simeq 0.8 B_{1,3}^{q} .
\end{aligned}
$$

The expressions for the Gegenbauer polynomials $C_{1}^{3 / 2}(t)$ and $C_{3}^{3 / 2}(t)$ can be found explicitly, for example, from Eqs. (A8) and (A10) in Ref. [54] with $\lambda=3 / 2$.

The related weak effective Hamiltonian $H_{\text {eff }}$ for the $B_{d(s)}^{0} \rightarrow J / \psi f_{q(s)}$ decays mentioned above can be written as [55]

\footnotetext{
${ }^{1}$ Because of charge conjugation invariance or conservation of vector current, the neutral scalar $\sigma$ and $f_{0}$ mesons cannot be produced through the vector current, which, consequently, results in the zero values of their vector decay constants, i.e., $f_{f_{q}}=f_{f_{s}}=0$.
} 


$$
H_{\text {eff }}=\frac{G_{F}}{\sqrt{2}}\left\{V_{c b}^{*} V_{c Q}\left[C_{1}(\mu) O_{1}^{c}(\mu)+C_{2}(\mu) O_{2}^{c}(\mu)\right]-V_{t b}^{*} V_{t Q}\left[\sum_{i=3}^{10} C_{i}(\mu) O_{i}(\mu)\right]\right\}+\text { H.c. }
$$

with the Fermi constant $G_{F}=1.16639 \times 10^{-5} \mathrm{GeV}^{-2}$, the light $Q=d$, s quark, and Wilson coefficients $C_{i}(\mu)$ at the renormalization scale $\mu$. The local four-quark operators $O_{i}(i=1, \ldots, 10)$ are written as

(1) current-current (tree) operators

$$
O_{1}^{c}=\left(\bar{Q}_{\alpha} c_{\beta}\right)_{V-A}\left(\bar{c}_{\beta} b_{\alpha}\right)_{V-A}, \quad O_{2}^{c}=\left(\bar{Q}_{\alpha} c_{\alpha}\right)_{V-A}\left(\bar{c}_{\beta} b_{\beta}\right)_{V-A}
$$

(2) QCD penguin operators

$$
\begin{array}{ll}
O_{3}=\left(\bar{Q}_{\alpha} b_{\alpha}\right)_{V-A} \sum_{q^{\prime}}\left(\bar{q}_{\beta}^{\prime} q_{\beta}^{\prime}\right)_{V-A}, & O_{4}=\left(\bar{Q}_{\alpha} b_{\beta}\right)_{V-A} \sum_{q^{\prime}}\left(\bar{q}_{\beta}^{\prime} q_{\alpha}^{\prime}\right)_{V-A}, \\
O_{5}=\left(\bar{Q}_{\alpha} b_{\alpha}\right)_{V-A} \sum_{q^{\prime}}\left(\bar{q}_{\beta}^{\prime} q_{\beta}^{\prime}\right)_{V+A}, & O_{6}=\left(\bar{Q}_{\alpha} b_{\beta}\right)_{V-A} \sum_{q^{\prime}}\left(\bar{q}_{\beta}^{\prime} q_{\alpha}^{\prime}\right)_{V+A} ;
\end{array}
$$

(3) electroweak penguin operators

$$
\begin{array}{rlrl}
O_{7} & =\frac{3}{2}\left(\bar{Q}_{\alpha} b_{\alpha}\right)_{V-A} \sum_{q^{\prime}} e_{q^{\prime}}\left(\bar{q}_{\beta}^{\prime} q_{\beta}^{\prime}\right)_{V+A}, & O_{8} & =\frac{3}{2}\left(\bar{Q}_{\alpha} b_{\beta}\right)_{V-A} \sum_{q^{\prime}} e_{q^{\prime}}\left(\bar{q}_{\beta}^{\prime} q_{\alpha}^{\prime}\right)_{V+A}, \\
O_{9}=\frac{3}{2}\left(\bar{Q}_{\alpha} b_{\alpha}\right)_{V-A} \sum_{q^{\prime}} e_{q^{\prime}}\left(\bar{q}_{\beta}^{\prime} q_{\beta}^{\prime}\right)_{V-A}, & O_{10}=\frac{3}{2}\left(\bar{Q}_{\alpha} b_{\beta}\right)_{V-A} \sum_{q^{\prime}} e_{q^{\prime}}\left(\bar{q}_{\beta}^{\prime} q_{\alpha}^{\prime}\right)_{V-A},
\end{array}
$$

with the notations $\left(\bar{q}^{\prime} q^{\prime}\right)_{V \pm A}=\bar{q}^{\prime} \gamma_{\mu}\left(1 \pm \gamma_{5}\right) q^{\prime}$. The index $q^{\prime}$ in the summation of the above operators runs through $u$, $d, s, c$, and $b$. The standard combinations $a_{i}$ of Wilson coefficients are defined as follows,

$$
\begin{aligned}
& a_{1}=C_{2}+\frac{C_{1}}{3}, \quad a_{2}=C_{1}+\frac{C_{2}}{3}, \\
& a_{i}=C_{i}+\frac{C_{i \pm 1}}{3}(i=3-10),
\end{aligned}
$$

where the upper (lower) sign applies, when $i$ is odd (even). It should be mentioned that, similar to $B \rightarrow J / \psi V$ decays [40], the NLO Wilson coefficients $C_{i}(i=1, \ldots, 10)$ and the strong coupling constant $\alpha_{s}$ at two-loop level with $\Lambda_{\mathrm{QCD}}^{(5)}=$ $0.225 \mathrm{GeV}$ [55] are adopted in the calculations of the $B_{d(s)}^{0} \rightarrow J / \psi f_{q(s)}$ decay amplitudes.

As for the decay amplitudes of $B_{d(s)}^{0} \rightarrow J / \psi f_{q(s)}$, we adopt $F_{f e}$ and $M_{n f e}$ to stand for the contributions of factorizable emission and nonfactorizable emission diagrams from $(V-A)(V-A)$ operators. The explicit expressions of these two Feynman amplitudes $F_{f e}$ and $M_{n f e}$ can be obtained by replacing the distribution amplitudes $\phi_{\omega(\phi)}$ and $\phi_{\omega(\phi)}^{s, t}$ in the $B_{d(s)}^{0} \rightarrow[J / \psi \omega(\phi)]_{L}$ mode $(L$ stands for longitudinal polarization), i.e., Eqs. (37) and (40) in [40], with those $\phi_{f_{q(s)}}$ and $\phi_{f_{q(s)}^{S, T}}$ correspondingly. Meanwhile, the masses of the light mesons should be replaced correspondingly too. Therefore, for simplicity, we do not present the factorization formulas of $F_{f e}$ and $M_{n f e}$ for the $B_{d(s)} \rightarrow$ $J / \psi f_{q(s)}$ decays in this work. The readers can refer to Ref. [40] for detail.

By taking various contributions from the relevant Feynman diagrams into consideration, the total decay amplitudes for $B_{d(s)}^{0} \rightarrow J / \psi f_{q(s)}$ channels are given as

$$
\begin{aligned}
& \mathcal{A}\left(B_{d(s)}^{0} \rightarrow J / \psi f_{q(s)}\right) \\
& =F_{f e} f_{J / \psi}\left\{V_{c b}^{*} V_{c d(s)} \tilde{a}_{2}-V_{t b}^{*} V_{t d(s)}\left(\tilde{a}_{3}+\tilde{a}_{5}+\tilde{a}_{7}+\tilde{a}_{9}\right)\right\} \\
& \quad+M_{n f e}\left\{V_{c b}^{*} V_{c d(s)} C_{2}-V_{t b}^{*} V_{t d(s)}\left(C_{4}-C_{6}-C_{8}+C_{10}\right)\right\},
\end{aligned}
$$

where $\tilde{a}_{i}$ stands for the effective Wilson coefficients that include the contributions arising from the vertex corrections at NLO level. The explicit expressions of $\tilde{a}_{i}$ can be found in the Appendix.

\section{NUMERICAL RESULTS AND DISCUSSIONS}

We present the theoretical predictions about the interesting observables such as $C P$-averaged branching ratios and $C P$-violating asymmetries for those considered $B_{d, s}^{0} \rightarrow$ $J / \psi \sigma\left(f_{0}\right)$ decay modes in the PQCD approach. In numerical calculations, central values of the input parameters are used implicitly unless otherwise stated.

The masses (in units of $\mathrm{GeV}$ ) and $B_{d, s}^{0}$ meson lifetime (in ps) are taken from Refs. [4,53], 


$$
\begin{array}{lcccc}
m_{W}=80.41, & m_{B}=5.28, & m_{B_{s}}=5.37, & m_{b}=4.8, & m_{f_{q}}=0.99 ; \\
m_{f_{s}}=1.02, & m_{c}=1.5, & m_{J / \psi}=3.097, & \tau_{B_{d}}=1.520, & \tau_{B_{s}}=1.509 .
\end{array}
$$

For the CKM matrix elements, we adopt the Wolfenstein parametrization up to corrections of $\mathcal{O}\left(\lambda^{5}\right)$ and the updated parameters $A=0.836, \lambda=0.22453, \bar{\rho}=0.122_{-0.017}^{+0.018}$, and $\bar{\eta}=0.355_{-0.011}^{+0.012}$ [4].

By employing those decay amplitudes, i.e., Eqs. (9)-(12) and Eq. (25), the formulas of branching ratios for the considered $B_{d, s}^{0} \rightarrow J / \psi \sigma\left(f_{0}\right)$ decays can be written as

$$
\begin{aligned}
\operatorname{BR}\left(B_{d, s}^{0} \rightarrow J / \psi \sigma\left(f_{0}\right)\right) & \equiv \tau_{B_{d(s)}^{0}} \cdot \Gamma\left(B_{d, s}^{0} \rightarrow J / \psi \sigma\left(f_{0}\right)\right) \\
& =\tau_{B_{d(s)}^{0}} \cdot \frac{G_{F}^{2} m_{B_{d(s)}^{0}}^{7}}{16 \pi} \cdot \Phi_{\sigma, f_{0}}^{d, s} \cdot\left|\mathcal{A}\left(B_{d, s}^{0} \rightarrow J / \psi \sigma\left(f_{0}\right)\right) / m_{B_{d(s)}^{0}}^{2}\right|^{2},
\end{aligned}
$$

where $\tau_{B_{d(s)}^{0}}$ is the lifetime of $B_{d(s)}^{0}$ meson and $\Phi_{\sigma, f_{0}}^{d, s}$ stands for the phase space factors of $B_{d, s}^{0} \rightarrow J / \psi \sigma\left(f_{0}\right)$ decays,

$$
\Phi_{\sigma\left(f_{0}\right)}^{d} \equiv \Phi\left(m_{J / \psi} / m_{B_{d}^{0}}, m_{\sigma\left(f_{0}\right)} / m_{B_{d}^{0}}\right), \quad \Phi_{\sigma\left(f_{0}\right)}^{s} \equiv \Phi\left(m_{J / \psi} / m_{B_{s}^{0}}, m_{\sigma\left(f_{0}\right)} / m_{B_{s}^{0}}\right),
$$

with $\Phi(x, y) \equiv \sqrt{\left[1-(x+y)^{2}\right]\left[1-(x-y)^{2}\right]}[28], m_{\sigma}=$ $0.5 \mathrm{GeV}$, and $m_{f_{0}}=0.98 \mathrm{GeV}$.

As discussed in the literature, up to now, the mixing angle $\phi_{f}$ between the mixtures of $\sigma$ and $f_{0}$ could not be determined definitely yet and is still in controversy. Various values and/or ranges have been analyzed; e.g., see Ref. [28,48] and references contained therein. However, based on lots of measurements via resonance investigations on the $B_{d, s}^{0} \rightarrow J / \psi \sigma\left(f_{0}\right)$ decays as presented in Eqs. (1)(4), it may be more interesting to consider the dependence of the $C P$-averaged branching ratios of $B_{d, s}^{0} \rightarrow$ $J / \psi \sigma / f_{0}\left(\rightarrow \pi^{+} \pi^{-}\right)$with the angle $\phi_{f}$ in the PQCD approach, which would hint effectively at the acceptable value of $\phi_{f}$ in this work. Certainly, different from the corresponding quasitwo-body decays [32], the $\sigma / f_{0} \rightarrow$ $\pi^{+} \pi^{-}$decay rate is regarded as an input in this work.

It is noted that the $f_{0}$ is an elusive object that decays largely into $\pi^{+} \pi^{-}$but also decays into $K^{+} K^{-}$. By combining the $B A B A R$ measurements about the $B \rightarrow$ $K K K, K \pi \pi$ decays and the BES measurements about $\psi(2 S) \rightarrow \gamma \chi_{c 0}\left(\rightarrow f_{0} f_{0}\right)$ decays with either both $f_{0}$ decaying into $\pi^{+} \pi^{-}$or one into $\pi^{+} \pi^{-}$and the other into $K^{+} K^{-}$pairs [56-59], the average of these two measurements could give [6]

$$
\mathcal{R} \equiv \frac{\mathcal{B}\left(f_{0} \rightarrow K^{+} K^{-}\right)}{\mathcal{B}\left(f_{0} \rightarrow \pi^{+} \pi^{-}\right)}=0.35_{-0.14}^{+0.15}
$$

which results in the following branching ratios explicitly:

$$
\begin{aligned}
\mathcal{B}\left(f_{0} \rightarrow \pi^{+} \pi^{-}\right) & =0.45_{-0.05}^{+0.07}, \\
\mathcal{B}\left(f_{0} \rightarrow K^{+} K^{-}\right) & =0.16_{-0.05}^{+0.04}
\end{aligned}
$$

by employing the formulas $\mathcal{B}\left(f_{0} \rightarrow \pi^{+} \pi^{-}\right)=\frac{2}{4 \mathcal{R}+3}$ and $\mathcal{B}\left(f_{0} \rightarrow K^{+} K^{-}\right)=\frac{2 \mathcal{R}}{4 \mathcal{R}+3}$ [28]. Here, the dominance of $f_{0}$ decaying into $\pi \pi$ and $K K$ is assumed, and the only other decays are also assumed to $\pi^{0} \pi^{0}$, half of the $\pi^{+} \pi^{-}$rate, and to $K^{0} \bar{K}^{0}$, taken equal to $K^{+} K^{-}$. For the $\sigma$ meson, it is assumed that the only decays are into two pions. Then, following from the isospin Clebsch-Gordan coefficients, the $\sigma \rightarrow \pi^{+} \pi^{-}$ decay rate could be obtained as $\frac{2}{3}$. In order to estimate the uncertainties from $\sigma \rightarrow \pi^{+} \pi^{-}$decay, the variations with $10 \%$ of the central value, i.e., $\mathcal{B}\left(\sigma \rightarrow \pi^{+} \pi^{-}\right) \simeq 0.67 \pm 0.07$, are taken into account in the following estimations.

Therefore, armed with $\mathcal{B}\left(f_{0} \rightarrow \pi^{+} \pi^{-}\right)$and $\mathcal{B}\left(\sigma \rightarrow \pi^{+} \pi^{-}\right)$, the $B_{d, s}^{0} \rightarrow J / \psi \sigma / f_{0}\left(\rightarrow \pi^{+} \pi^{-}\right)$decay rates varying with the mixing angle $\phi_{f}$ could be further written theoretically as [60]

$$
\begin{aligned}
\mathrm{BR}\left(B_{d}^{0} \rightarrow J / \psi \sigma, \sigma \rightarrow \pi^{+} \pi^{-}\right) & \equiv \mathrm{BR}\left(B_{d}^{0} \rightarrow J / \psi \sigma\right) \mathcal{B}\left(\sigma \rightarrow \pi^{+} \pi^{-}\right) \\
& \propto \tau_{B_{d}^{0}} \cdot \Phi_{\sigma}^{d} \cdot m_{B_{d}^{0}}^{7} \cdot\left|\mathcal{A}\left(B_{d}^{0} \rightarrow J / \psi f_{q}\right) / m_{B_{d}^{0}}^{2}\right|^{2} \cdot \cos ^{2} \phi_{f}, \\
\operatorname{BR}\left(B_{d}^{0} \rightarrow J / \psi f_{0}, f_{0} \rightarrow \pi^{+} \pi^{-}\right) & \equiv \mathrm{BR}\left(B_{d}^{0} \rightarrow J / \psi f_{0}\right) \mathcal{B}\left(f_{0} \rightarrow \pi^{+} \pi^{-}\right) \\
& \propto \tau_{B_{d}^{0}} \cdot \Phi_{f_{0}}^{d} \cdot m_{B_{d}^{0}}^{7} \cdot\left|\mathcal{A}\left(B_{d}^{0} \rightarrow J / \psi f_{q}\right) / m_{B_{d}^{0}}^{2}\right|^{2} \cdot \sin ^{2} \phi_{f} \\
\operatorname{BR}\left(B_{s}^{0} \rightarrow J / \psi \sigma, \sigma \rightarrow \pi^{+} \pi^{-}\right) & \equiv \mathrm{BR}\left(B_{s}^{0} \rightarrow J / \psi \sigma\right) \mathcal{B}\left(\sigma \rightarrow \pi^{+} \pi^{-}\right) \\
& \propto \tau_{B_{s}^{0}} \cdot \Phi_{\sigma}^{s} \cdot m_{B_{s}^{0}}^{7} \cdot\left|\mathcal{A}\left(B_{s}^{0} \rightarrow J / \psi f_{s}\right) / m_{B_{s}^{0}}^{2}\right|^{2} \cdot \sin ^{2} \phi_{f},
\end{aligned}
$$



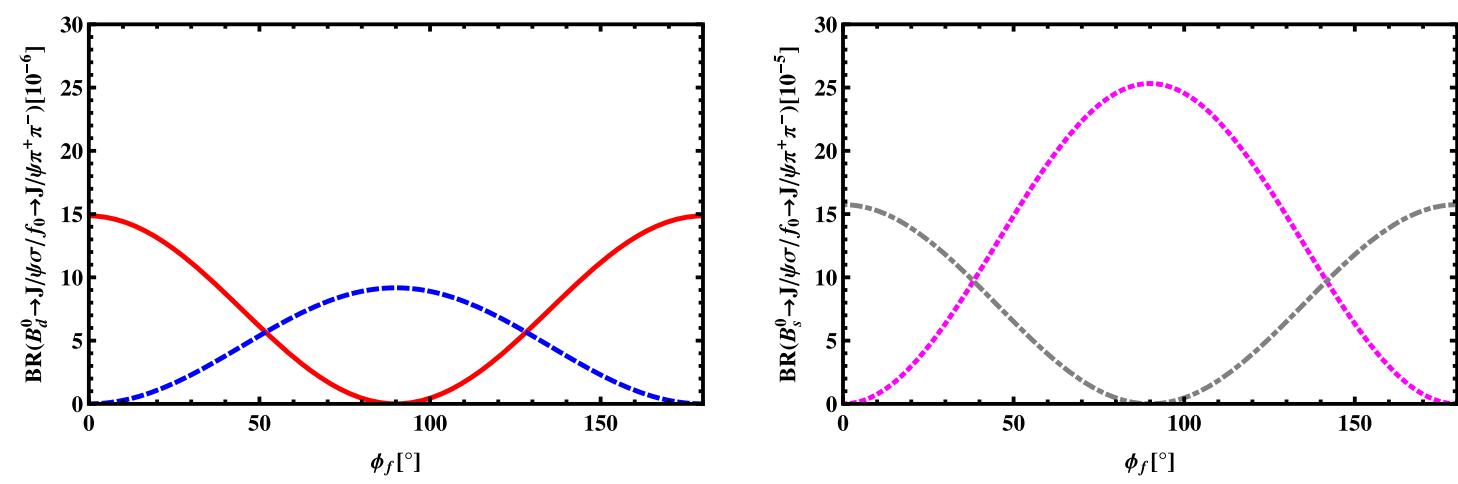

FIG. 2. Dependence on the mixing angle $\phi_{f}$ of the central values for $\operatorname{BR}\left(B_{d, s}^{0} \rightarrow J / \psi \sigma / f_{0} \rightarrow J / \psi \pi^{+} \pi^{-}\right)$in the PQCD approach: The red solid (blue dashed) line corresponds to the $B_{d}^{0} \rightarrow J / \psi \sigma\left(\rightarrow \pi^{+} \pi^{-}\right)\left[B_{d}^{0} \rightarrow J / \psi f_{0}\left(\rightarrow \pi^{+} \pi^{-}\right)\right]$decay, and the magenta dotted (gray dotdashed) line corresponds to the $B_{s}^{0} \rightarrow J / \psi \sigma\left(\rightarrow \pi^{+} \pi^{-}\right)\left[B_{s}^{0} \rightarrow J / \psi f_{0}\left(\rightarrow \pi^{+} \pi^{-}\right)\right]$decay, respectively.

$$
\begin{aligned}
\operatorname{BR}\left(B_{s}^{0} \rightarrow J / \psi f_{0}, f_{0} \rightarrow \pi^{+} \pi^{-}\right) & \equiv \operatorname{BR}\left(B_{s}^{0} \rightarrow J / \psi f_{0}\right) \mathcal{B}\left(f_{0} \rightarrow \pi^{+} \pi^{-}\right) \\
& \propto \tau_{B_{s}^{0}} \cdot \Phi_{f_{0}}^{s} \cdot m_{B_{s}^{0}}^{7} \cdot\left|\mathcal{A}\left(B_{s}^{0} \rightarrow J / \psi f_{s}\right) / m_{B_{s}^{0}}^{2}\right|^{2} \cdot \cos ^{2} \phi_{f} .
\end{aligned}
$$

By employing the decay amplitudes and the hadronic inputs, we plot the $C P$-averaged branching ratios in the PQCD approach at the known NLO level of $B_{d, s}^{0} \rightarrow J / \psi \sigma /$ $f_{0}\left(\rightarrow \pi^{+} \pi^{-}\right)$decays depending on the angle $\phi_{f}$, which can be seen explicitly in Fig. 2. Here, the central values of the relevant branching ratios varying with $\phi_{f}$ are presented for clarification. By comparing with the data as shown in Eqs. (1)-(4), one can easily observe the overall consistency between experiment and theory of $\operatorname{BR}\left(B_{d, s}^{0} \rightarrow J / \psi \sigma / f_{0}(\rightarrow\right.$ $\left.\pi^{+} \pi^{-}\right)$) around $\phi_{f} \approx 25^{\circ}$ with a twofold ambiguity from
Fig. 2. Frankly speaking, this twofold ambiguity cannot be resolved in these considered $B_{d, s}^{0} \rightarrow J / \psi \sigma\left(f_{0}\right)$ decays because there are no any interferences between the final states $J / \psi f_{q}$ and $J / \psi f_{s}$. That means it tends to be resolved through the studies of other $B \rightarrow M \sigma\left(f_{0}\right)$ decays with $M$ denoting the open-charmed or light hadrons, once the related measurements are available with high precision.

Then, within theoretical uncertainties, the NLO PQCD predictions of $\operatorname{BR}\left(B_{d, s}^{0} \rightarrow J / \psi \sigma\left(f_{0}\right), \sigma\left(f_{0}\right) \rightarrow \pi^{+} \pi^{-}\right)$at $\phi_{f} \approx 25^{\circ}$ can be read as follows:

$$
\begin{aligned}
\mathrm{BR}\left(B_{d}^{0} \rightarrow J / \psi \sigma, \sigma \rightarrow \pi^{+} \pi^{-}\right) & =1.22_{-0.29}^{+0.41}\left(\omega_{B}\right)_{-0.17}^{+0.19}\left(f_{J / \psi}\right)_{-0.29}^{+0.34}\left(B_{i}^{q}\right)_{-0.21}^{+0.13}\left(a_{t}\right)_{-0.12}^{+0.12}\left(\mathcal{B}_{\sigma}\right) \times 10^{-5} \\
& =1.22_{-0.51}^{+0.60} \times 10^{-5}, \\
\mathrm{BR}\left(B_{s}^{0} \rightarrow J / \psi f_{0}, f_{0} \rightarrow \pi^{+} \pi^{-}\right) & =1.30_{-0.33}^{+0.50}\left(\omega_{B}\right)_{-0.18}^{+0.21}\left(f_{J / \psi}\right)_{-0.27}^{+0.30}\left(B_{i}^{s}\right)_{-0.23}^{+0.19}\left(a_{t}\right)_{-0.14}^{+0.20}\left(\mathcal{B}_{f_{0}}\right) \times 10^{-4} \\
& =1.30_{-0.53}^{+0.68} \times 10^{-4} ; \\
\mathrm{BR}\left(B_{d}^{0} \rightarrow J / \psi f_{0}, f_{0} \rightarrow \pi^{+} \pi^{-}\right) & =1.64_{-0.39}^{+0.54}\left(\omega_{B}\right)_{-0.23}^{+0.26}\left(f_{J / \psi}\right)_{-0.39}^{+0.46}\left(B_{i}^{q}\right)_{-0.28}^{+0.17}\left(a_{t}\right)_{-0.18}^{+0.25}\left(\mathcal{B}_{f_{0}}\right) \times 10^{-6} \\
& =1.64_{-0.69}^{+0.81} \times 10^{-6}, \\
\operatorname{BR}\left(B_{s}^{0} \rightarrow J / \psi \sigma, \sigma \rightarrow \pi^{+} \pi^{-}\right) & =4.56_{-1.16}^{+1.74}\left(\omega_{B}\right)_{-0.63}^{+0.71}\left(f_{J / \psi}\right)_{-0.93}^{+1.06}\left(B_{i}^{s}\right)_{-0.80}^{+0.66}\left(a_{t}\right)_{-0.45}^{+0.46}\left(\mathcal{B}_{\sigma}\right) \times 10^{-5} \\
& =4.56_{-1.86}^{+2.30} \times 10^{-5} .
\end{aligned}
$$

The dominant errors are induced by the shape parameter $\omega_{B}=0.40 \pm 0.04\left(\omega_{B}=0.50 \pm 0.05\right) \mathrm{GeV}$ for the $B_{d}^{0}\left(B_{s}^{0}\right)$ meson, the decay constant $f_{J / \psi}=0.405 \pm 0.014 \mathrm{GeV}$ for the $J / \psi$ meson, the Gegenbauer moments $B_{i}^{q, s}$ [see Eq. (19)] in the leading-twist light-cone distribution amplitude of light scalar $f_{q, s}$ states, and the branching ratios $\mathcal{B}_{\sigma / f_{0} \rightarrow \pi^{+} \pi^{-}}$, respectively. Furthermore, we also investigate the higher order contributions simply through exploring the variation of the running hard scale $t_{\max }$, i.e., from $0.8 t$ to $1.2 t$ (not changing $1 / b_{i}, i=1,2,3$ ), in the hard kernel, which has been counted into one of the sources of theoretical uncertainties. In every second line of 
the above equations, various errors have been added in quadrature.

It is worthwhile to stress that, within still large uncertainties, the NLO PQCD predictions about the $B_{d}^{0} \rightarrow$ $J / \psi \sigma\left(\rightarrow \pi^{+} \pi^{-}\right)$and $B_{d, s}^{0} \rightarrow J / \psi f_{0}\left(\rightarrow \pi^{+} \pi^{-}\right)$decay rates are generally consistent with the current data or upper limits, except for the seemingly challenging $B_{s}^{0} \rightarrow J / \psi \sigma(\rightarrow$ $\pi^{+} \pi^{-}$) one. Nevertheless, roughly speaking, the theoretical prediction of $\operatorname{BR}\left(B_{s}^{0} \rightarrow J / \psi \sigma\left(\rightarrow \pi^{+} \pi^{-}\right)\right)$could agree with the current upper limits within $3 \sigma$ (not to be confused with the $\sigma$ meson) standard deviations. Of course, more relevant studies are demanded theoretically and experimentally.

In order to find more evidences for the consistency between theory and experiment under the assumption of $\sigma-f_{0}$ mixing in the conventional two-quark structure, it is better for us to study the relative ratios of the above-mentioned branching ratios over those of the referenced channels such as the preferred $\operatorname{BR}\left(B_{s}^{0} \rightarrow J /\right.$ $\left.\psi \phi\left(\rightarrow K^{+} K^{-}\right)\right)$, because the effects induced by the uncertainties of nonperturbative inputs are expected to be canceled to a great extent. This cancellation can also be easily observed in the quantities such as $C P$-violating asymmetries that are clarified later. Therefore, following Eqs. (5)-(7), the relative ratio $R_{f_{0} / \phi}^{\mathrm{Th}}(\pi)$ in the PQCD approach at NLO accuracy could be easily obtained as

$$
\begin{aligned}
R_{f_{0} / \phi}^{\mathrm{Th}}(\pi) & \left.\equiv \frac{\mathrm{BR}\left(B_{s}^{0} \rightarrow J / \psi f_{0}\right) \mathcal{B}\left(f_{0} \rightarrow \pi^{+} \pi^{-}\right)}{\operatorname{BR}\left(B_{s}^{0} \rightarrow J / \psi \phi\right) \mathcal{B}\left(\phi \rightarrow K^{+} K^{-}\right)}\right|_{\mathrm{PQCD}} \\
& =0.258_{-0.041}^{+0.032},
\end{aligned}
$$

and

$$
\left.\frac{\mathrm{BR}\left(B_{s}^{0} \rightarrow J / \psi f_{0}, f_{0} \rightarrow \pi^{+} \pi^{-}\right)}{\operatorname{BR}\left(B_{s}^{0} \rightarrow J / \psi \phi\right)}\right|_{\mathrm{PQCD}}=0.126_{-0.020}^{+0.017},
$$

assisted with the available values $\left.\operatorname{BR}\left(B_{s}^{0} \rightarrow J / \psi \phi\right)\right|_{\mathrm{PQCD}}=$ $1.02_{-0.30}^{+0.36} \times 10^{-3}[40]$ and $\mathcal{B}\left(\phi \rightarrow K^{+} K^{-}\right)=0.492 \pm 0.005$ [4]. These two ratios are found to agree well with the measurements as shown in Eqs. (6) and (7).

Furthermore, as reported by the LHCb Collaboration, the latest values of $\operatorname{BR}\left(B_{d}^{0} \rightarrow J / \psi \rho^{0}, \rho^{0} \rightarrow \pi^{+} \pi^{-}\right)$and $\operatorname{BR}\left(B_{d}^{0} \rightarrow J / \psi \sigma, \sigma \rightarrow \pi^{+} \pi^{-}\right)$are as follows [7],

$$
\begin{aligned}
& \mathrm{BR}\left(B_{d}^{0} \rightarrow J / \psi \rho^{0}, \rho^{0} \rightarrow \pi^{+} \pi^{-}\right)=2.50_{-0.18}^{+0.21} \times 10^{-5}, \\
& \mathrm{BR}\left(B_{d}^{0} \rightarrow J / \psi \sigma, \sigma \rightarrow \pi^{+} \pi^{-}\right)=0.88_{-0.16}^{+0.12} \times 10^{-5} .
\end{aligned}
$$

Then the relative ratio of these two branching ratios could be derived analogously as
$R_{\sigma / \rho} \equiv \frac{\mathrm{BR}\left(B_{d}^{0} \rightarrow J / \psi \sigma, \sigma \rightarrow \pi^{+} \pi^{-}\right)}{\operatorname{BR}\left(B_{d}^{0} \rightarrow J / \psi \rho^{0}, \rho^{0} \rightarrow \pi^{+} \pi^{-}\right)}=0.352_{-0.042}^{+0.017}$,

It is commented that, based on the isospin conservation in the strong interactions, the branching ratio of $\rho^{0} \rightarrow \pi^{+} \pi^{-}$is about $100 \%$ [4]. Therefore, by combining with the available prediction $\left.\mathrm{BR}\left(B_{d}^{0} \rightarrow J / \psi \rho^{0}\right)\right|_{\mathrm{PQCD}}=2.7_{-0.7}^{+1.0} \times 10^{-5}$ [40] and Eq. (35), the corresponding ratio predicted theoretically in the PQCD approach can be read as

$$
\begin{aligned}
R_{\sigma / \rho}^{\mathrm{Th}}(\pi) & \left.\equiv \frac{\mathrm{BR}\left(B_{d}^{0} \rightarrow J / \psi \sigma\right) \mathcal{B}\left(\sigma \rightarrow \pi^{+} \pi^{-}\right)}{\operatorname{BR}\left(B_{d}^{0} \rightarrow J / \psi \rho^{0}\right) \mathcal{B}\left(\rho^{0} \rightarrow \pi^{+} \pi^{-}\right)}\right|_{\mathrm{PQCD}} \\
& =0.452_{-0.097}^{+0.040},
\end{aligned}
$$

which is basically consistent with that, see Eq. (43), extracted from the $\mathrm{LHCb}$ measurement within large errors. It is clearly observed that the PQCD predicted branching ratios and the relevant ratios of $B_{d(s)}^{0} \rightarrow J / \psi \sigma\left(f_{0}\right)$ $\left(\rightarrow \pi^{+} \pi^{-}\right)$decays with the mixing angle $\phi_{f}$ around $25^{\circ}$ indeed agree with the corresponding measurements within uncertainties. It is interesting to note that these predictions are also consistent with those already presented in the literature [28,32].

Similarly, the ratios $R_{f_{0} / \rho}^{\mathrm{Th}}(\pi)$ and $R_{\sigma / \phi}^{\mathrm{Th}}(\pi)$ in the PQCD approach could be predicted as

$$
\begin{aligned}
R_{f_{0} / \rho}^{\mathrm{Th}}(\pi) & \left.\equiv \frac{\mathrm{BR}\left(B_{d}^{0} \rightarrow J / \psi f_{0}\right) \mathcal{B}\left(f_{0} \rightarrow \pi^{+} \pi^{-}\right)}{\mathrm{BR}\left(B_{d}^{0} \rightarrow J / \psi \rho^{0}\right) \mathcal{B}\left(\rho^{0} \rightarrow \pi^{+} \pi^{-}\right)}\right|_{\mathrm{PQCD}} \\
& =0.061_{-0.013}^{+0.005}, \\
R_{\sigma / \phi}^{\mathrm{Th}}(\pi) & \left.\equiv \frac{\mathrm{BR}\left(B_{s}^{0} \rightarrow J / \psi \sigma\right) \mathcal{B}\left(\sigma \rightarrow \pi^{+} \pi^{-}\right)}{\mathrm{BR}\left(B_{s}^{0} \rightarrow J / \psi \phi\right) \mathcal{B}\left(\phi \rightarrow K^{+} K^{-}\right)}\right|_{\mathrm{PQCD}} \\
& =0.090_{-0.014}^{+0.010},
\end{aligned}
$$

which are expected to be examined in the future measurements, even if the $B_{s}^{0} \rightarrow J / \psi \sigma\left(\sigma \rightarrow \pi^{+} \pi^{-}\right)$decay rate highly supersedes the current upper limit set by the LHCb Collaboration.

From the above results, one can see that most of our PQCD predictions on $C P$-averaged branching ratios and relevantly relative ratios of $B_{d, s}^{0} \rightarrow J / \psi \sigma / f_{0}\left(\rightarrow \pi^{+} \pi^{-}\right)$up to NLO precision agree well with the existing experimental measurements within uncertainties at $\phi_{f}$ around $25^{\circ}$. Therefore, the branching ratios of the decays $B_{d, s}^{0} \rightarrow$ $J / \psi \sigma\left(f_{0}\right)$ under consideration in the PQCD approach are presented within errors as follows,

(i) for $\bar{b} \rightarrow \bar{d}$ decay channels,

$$
\mathrm{BR}\left(B_{d}^{0} \rightarrow J / \psi \sigma\right)=1.83_{-0.43}^{+0.61}\left(\omega_{B}\right)_{-0.25}^{+0.29}\left(f_{J / \psi}\right)_{-0.44}^{+0.51}\left(B_{i}^{q}\right)_{-0.31}^{+0.19}\left(a_{t}\right)\left[1.83_{-0.73}^{+0.87}\right] \times 10^{-5},
$$




$$
\mathrm{BR}\left(B_{d}^{0} \rightarrow J / \psi f_{0}\right)=3.64_{-0.86}^{+1.21}\left(\omega_{B}\right)_{-0.51}^{+0.57}\left(f_{J / \psi}\right)_{-0.88}^{+1.02}\left(B_{i}^{q}\right)_{-0.62}^{+0.37}\left(a_{t}\right)\left[3.64_{-1.47}^{+1.72}\right] \times 10^{-6}
$$

(ii) for $\bar{b} \rightarrow \bar{s}$ decay channels,

$$
\begin{aligned}
& \mathrm{BR}\left(B_{s}^{0} \rightarrow J / \psi \sigma\right)=6.83_{-1.74}^{+2.61}\left(\omega_{B}\right)_{-0.95}^{+1.07}\left(f_{J / \psi}\right)_{-1.40}^{+1.58}\left(B_{i}^{s}\right)_{-1.20}^{+0.99}\left(a_{t}\right)\left[6.83_{-2.71}^{+3.38}\right] \times 10^{-5}, \\
& \mathrm{BR}\left(B_{s}^{0} \rightarrow J / \psi f_{0}\right)=2.89_{-0.73}^{+1.11}\left(\omega_{B}\right)_{-0.40}^{+0.46}\left(f_{J / \psi}\right)_{-0.58}^{+0.67}\left(B_{i}^{s}\right)_{-0.50}^{+0.42}\left(a_{t}\right)\left[2.89_{-1.13}^{+1.44}\right] \times 10^{-4},
\end{aligned}
$$

where, as shown in the square brackets, various errors of the numerical results have also been added in quadrature. One can observe that the decay rates for the $\bar{b} \rightarrow \bar{s}$ transition processes, i.e., $B_{s}^{0} \rightarrow J / \psi \sigma\left(f_{0}\right)$, are generally much larger than those for the $\bar{b} \rightarrow \bar{d}$ transition ones, i.e., $B_{d}^{0} \rightarrow J / \psi \sigma\left(f_{0}\right)$. This is due to the CKM hierarchy for two kinds of processes: the CKM factors $V_{c b} V_{c s}$ in $b \rightarrow s$ are about four times larger than the $V_{c b} V_{c d}$ for $b \rightarrow d$ process, and the different factors $\sin ^{2} \phi_{f}$ or $\cos ^{2} \phi_{f}$ from the mixtures of $\sigma$ and $f_{0}$ mesons. The remanent but small differences arise from the $\mathrm{SU}(3)$ symmetry breaking effects in the hadronic parameters, such as decay constants, mesonic masses, distribution amplitudes, etc. It is easily seen that our NLO PQCD predicted branching ratios of the $B_{d, s}^{0} \rightarrow$ $J / \psi \sigma\left(f_{0}\right)$ decays around $\phi_{f} \approx 25^{\circ}$ are generally consistent with those earlier predictions [25-29] as aforementioned in the introduction within still large uncertainties.

Based on those PQCD branching ratios as presented in the Eqs. (47)-(50), several interesting ratios could be derived as follows:

$$
\begin{aligned}
R_{\sigma f_{0}}^{d} & \left.\equiv \frac{\mathrm{BR}\left(B_{d}^{0} \rightarrow J / \psi \sigma\right)}{\mathrm{BR}\left(B_{d}^{0} \rightarrow J / \psi f_{0}\right)}\right|_{\mathrm{PQCD}}\left(\approx 5.03_{-0.01}^{+0.02}\right)=\frac{\Phi_{\sigma}^{d}}{\Phi_{f_{0}}^{d}} \cdot \cot ^{2} \phi_{f}, \\
R_{f_{0} \sigma}^{s} \equiv & \left.\equiv \frac{\mathrm{BR}\left(B_{s}^{0} \rightarrow J / \psi f_{0}\right)}{\mathrm{BR}\left(B_{s}^{0} \rightarrow J / \psi \sigma\right)}\right|_{\mathrm{PQCD}}\left(\approx 4.23_{-0.00}^{+0.03}\right)=\frac{\Phi_{f_{0}}^{s}}{\Phi_{\sigma}^{s}} \cdot \cot ^{2} \phi_{f} \\
R_{s d}^{\sigma} & \left.\equiv \frac{\mathrm{BR}\left(B_{s}^{0} \rightarrow J / \psi \sigma\right)}{\mathrm{BR}\left(B_{d}^{0} \rightarrow J / \psi \sigma\right)}\right|_{\mathrm{PQCD}}\left(\approx 3.73_{-0.17}^{+0.27}\right) \\
& =\frac{\tau_{B_{s}^{0}}}{\tau_{B_{d}^{0}}} \cdot\left(\frac{m_{B_{s}^{0}}}{m_{B_{d}^{0}}}\right)^{7} \cdot \frac{\Phi_{\sigma}^{s}}{\Phi_{\sigma}^{d}} \cdot \frac{\left|\mathcal{A}\left(B_{s}^{0} \rightarrow J / \psi f_{s}\right) / m_{B_{s}^{0}}^{2}\right|^{2}}{\left|\mathcal{A}\left(B_{d}^{0} \rightarrow J / \psi f_{q}\right) / m_{B_{d}^{0}}^{2}\right|^{2}} \cdot \tan ^{2} \phi_{f}, \\
R_{s d}^{f_{0}} & \left.\equiv \frac{\mathrm{BR}\left(B_{s}^{0} \rightarrow J / \psi f_{0}\right)}{\mathrm{BR}\left(B_{d}^{0} \rightarrow J / \psi f_{0}\right)}\right|_{\mathrm{PQCD}}\left(\approx 79.43_{-3.45}^{+6.13}\right) \\
& =\frac{\tau_{B_{s}^{0}}^{0}}{\tau_{B_{d}^{0}}} \cdot\left(\frac{m_{B_{s}^{0}}}{m_{B_{d}^{0}}}\right)^{7} \cdot \frac{\Phi_{f_{0}}^{s}}{\Phi_{f_{0}}^{d}} \cdot \frac{\left|\mathcal{A}\left(B_{s}^{0} \rightarrow J / \psi f_{s}\right) / m_{B_{s}^{0}}^{2}\right|^{2}}{\left|\mathcal{A}\left(B_{d}^{0} \rightarrow J / \psi f_{q}\right) / m_{B_{d}^{0}}^{2}\right|^{2}} \cdot \cot ^{2} \phi_{f} .
\end{aligned}
$$

$$
\begin{aligned}
R_{d \sigma}^{s f_{0}} & \left.\equiv \frac{\operatorname{BR}\left(B_{s}^{0} \rightarrow J / \psi f_{0}\right)}{\operatorname{BR}\left(B_{d}^{0} \rightarrow J / \psi \sigma\right)}\right|_{\mathrm{PQCD}}\left(\approx 15.8_{-0.7}^{+1.2}\right) \\
& =\frac{\tau_{B_{s}^{0}}}{\tau_{B_{d}^{0}}} \cdot\left(\frac{m_{B_{s}^{0}}}{m_{B_{d}^{0}}}\right)^{7} \cdot \frac{\Phi_{f_{0}}^{s}}{\Phi_{\sigma}^{d}} \cdot \frac{\left|\mathcal{A}\left(B_{s}^{0} \rightarrow J / \psi f_{s}\right) / m_{B_{s}^{0}}^{2}\right|^{2}}{\left|\mathcal{A}\left(B_{d}^{0} \rightarrow J / \psi f_{q}\right) / m_{B_{d}^{0}}^{2}\right|^{2}} \\
R_{d f_{0}}^{s \sigma} & \left.\equiv \frac{\operatorname{BR}\left(B_{s}^{0} \rightarrow J / \psi \sigma\right)}{\operatorname{BR}\left(B_{d}^{0} \rightarrow J / \psi f_{0}\right)}\right|_{\mathrm{PQCD}}\left(\approx 18.7_{-0.9}^{+1.4}\right) \\
& =\frac{\tau_{B_{s}^{0}}}{\tau_{B_{d}^{0}}} \cdot\left(\frac{m_{B_{s}^{0}}}{m_{B_{d}^{0}}}\right)^{7} \cdot \frac{\Phi_{\sigma}^{s}}{\Phi_{f_{0}}^{d}} \cdot \frac{\left|\mathcal{A}\left(B_{s}^{0} \rightarrow J / \psi f_{s}\right) / m_{B_{s}^{0}}^{2}\right|^{2}}{\left|\mathcal{A}\left(B_{d}^{0} \rightarrow J / \psi f_{q}\right) / m_{B_{d}^{0}}^{2}\right|^{2}} .
\end{aligned}
$$

Then, some remarks are in order.

(a) It is interesting to note that the first two ratios $R_{\sigma f_{0}}^{d}$ and $R_{f_{0} \sigma}^{s}$ in the PQCD approach are almost invariant to the aforementioned various nonperturbative parameters, although the corresponding branching ratios show strong sensitivity to them. Again, the effects induced by various errors in the relevant branching ratios have been canceled significantly. Thus, as discussed in the literature, e.g., Refs. [24,29], these two relations could be utilized to extract the angle $\phi_{f}$ between $\sigma$ and $f_{0}$ mixing in the two-quark picture cleanly, because $R_{\sigma f_{0}}^{d}$ and $R_{f_{0} \sigma}^{s}$ are almost equal to $\cot ^{2} \phi_{f}$ with the almost definite values $\Phi_{\sigma}^{d} / \Phi_{f_{0}}^{d} \approx 1.095$ and $\Phi_{\sigma}^{s} / \Phi_{f_{0}}^{s} \approx 1.087$, respectively.

(b) As presented in the last two ratios, $R_{d \sigma}^{s f_{0}}$ and $R_{d f_{0}}^{s \sigma}$ are independent on the mixing angle $\phi_{f}$, and are of great interest to examine the SU(3) flavor symmetry breaking effects, if the penguin contributions are indeed tiny and negligible. To see more explicitly, these two ratios could be further derived by factoring out the related CKM matrix elements $V_{c s}$ and $V_{c d}$ correspondingly,

$$
\begin{aligned}
& R_{d \sigma}^{s f_{0}}=\frac{\tau_{B_{s}^{0}}}{\tau_{B_{d}^{0}}} \cdot\left(\frac{m_{B_{s}^{0}}}{m_{B_{d}^{0}}}\right)^{7} \cdot \frac{\Phi_{f_{0}}^{s}}{\Phi_{\sigma}^{d}} \cdot \frac{\left|V_{c s}\right|^{2}}{\left|V_{c d}\right|^{2}} \cdot \frac{\left|\mathcal{A}^{\prime}\left(B_{s}^{0} \rightarrow J / \psi f_{s}\right)\right|^{2}}{\left|\mathcal{A}^{\prime}\left(B_{d}^{0} \rightarrow J / \psi f_{q}\right)\right|^{2}}, \\
& R_{d f_{0}}^{s \sigma}=\frac{\tau_{B_{s}^{0}}}{\tau_{B_{d}^{0}}} \cdot\left(\frac{m_{B_{s}^{0}}}{m_{B_{d}^{0}}}\right)^{7} \cdot \frac{\Phi_{\sigma}^{s}}{\Phi_{f_{0}}^{d}} \cdot \frac{\left|V_{c s}\right|^{2}}{\left|V_{c d}\right|^{2}} \cdot \frac{\left|\mathcal{A}^{\prime}\left(B_{s}^{0} \rightarrow J / \psi f_{s}\right)\right|^{2}}{\left|\mathcal{A}^{\prime}\left(B_{d}^{0} \rightarrow J / \psi f_{q}\right)\right|^{2}},
\end{aligned}
$$


which consequently result in $\frac{\left|\mathcal{A}^{\prime}\left(B_{s}^{0} \rightarrow J / \psi f_{s}\right)\right|^{2}}{\left|\mathcal{A}^{\prime}\left(B_{d}^{0} \rightarrow J / \psi f_{q}\right)\right|^{2}} \approx 0.72$, deviating from unity about $30 \%$ roughly. Here, $\mathcal{A}^{\prime} \equiv \mathcal{A} / m_{B}^{2}$.

(c) In light of the above-mentioned two points, it seems more complicated that the entanglement of the SU(3) symmetry breaking effects and the information of mixing angle $\phi_{f}$ exhibits evidently in the middle two relations. Nevertheless, these two ratios could provide constraints supplementarily to either the former or the latter when one of them in the first two or last two ratios could be manifested definitely.

By the way, the mixing angle $\phi_{f}$ can also be constrained similarly from the ratios of the measured $B_{d}^{0} \rightarrow J / \psi \sigma$ and $B_{s}^{0} \rightarrow J / \psi f_{0}$ decays over the referenced $B_{d}^{0} \rightarrow J / \psi \rho^{0}$ and $B_{s}^{0} \rightarrow J / \psi \phi$ ones with high precision, respectively, but suffer probably from nonperturbative pollution induced by the hadronic parameters.

Now, let us turn to analyze the $C P$ violations of the $B_{d, s}^{0} \rightarrow J / \psi \sigma\left(f_{0}\right)$ decays in the PQCD approach at NLO accuracy. As for the $C P$-violating asymmetries for the $B_{d, s}^{0} \rightarrow J / \psi \sigma\left(f_{0}\right)$ decays, the effects of neutral $B_{d, s}^{0}-\bar{B}_{d, s}^{0}$ mixing should be taken into account. The $C P$-violating asymmetries of $B_{d, s}^{0}\left(\bar{B}_{d, s}^{0}\right) \rightarrow J / \psi \sigma\left(f_{0}\right)$ decays are time dependent and can be defined as

$$
\begin{aligned}
A_{\mathrm{CP}} & \equiv \frac{\Gamma\left(\bar{B}_{d, s}^{0}(\Delta t) \rightarrow f_{\mathrm{CP}}\right)-\Gamma\left(B_{d, s}^{0}(\Delta t) \rightarrow f_{\mathrm{CP}}\right)}{\Gamma\left(\bar{B}_{d, s}^{0}(\Delta t) \rightarrow f_{\mathrm{CP}}\right)+\Gamma\left(B_{d, s}^{0}(\Delta t) \rightarrow f_{\mathrm{CP}}\right)} \\
& =A_{\mathrm{CP}}^{\mathrm{dir}} \cos \left(\Delta m_{d, s} \Delta t\right)+A_{\mathrm{CP}}^{\mathrm{mix}} \sin \left(\Delta m_{d, s} \Delta t\right),
\end{aligned}
$$

where $\Delta m_{d, s}$ is the mass difference between the two $B_{d, s}^{0}$ mass eigenstates, $\Delta t=t_{\mathrm{CP}}-t_{\mathrm{tag}}$ is the time difference between the tagged $B_{d, s}^{0}\left(\bar{B}_{d, s}^{0}\right)$ and the accompanying $\bar{B}_{d, s}^{0}$ $\left(B_{d, s}^{0}\right)$ with opposite $b$ flavor decaying to the final $C P$ eigenstate $f_{\mathrm{CP}}$ at the time $t_{\mathrm{CP}}$. The direct and mixinginduced $C P$-violating asymmetries $A_{\mathrm{CP}}^{\mathrm{dir}}\left(\mathcal{C}_{f}\right)$ and $A_{\mathrm{CP}}^{\operatorname{mix}}\left(\mathcal{S}_{f}\right)$ can be written as

$A_{\mathrm{CP}}^{\mathrm{dir}} \equiv \mathcal{C}_{f}=\frac{\left|\lambda_{\mathrm{CP}}^{d, s}\right|^{2}-1}{1+\left|\lambda_{\mathrm{CP}}^{d, s}\right|^{2}}, \quad A_{\mathrm{CP}}^{\mathrm{mix}} \equiv \mathcal{S}_{f}=\frac{2 \operatorname{Im}\left(\lambda_{\mathrm{CP}}^{d, s}\right)}{1+\left|\lambda_{\mathrm{CP}}^{d, s}\right|^{2}}$,

with the $C P$-violating parameter $\lambda_{\mathrm{CP}}^{d, s}$,

$$
\lambda_{\mathrm{CP}}^{d, s} \equiv \eta_{f} \frac{V_{t b}^{*} V_{t d(s)}}{V_{t b} V_{t d(s)}^{*}} \cdot \frac{\left\langle f_{\mathrm{CP}}\left|H_{\mathrm{eff}}\right| \bar{B}_{d, s}^{0}\right\rangle}{\left\langle f_{\mathrm{CP}}\left|H_{\mathrm{eff}}\right| B_{d, s}^{0}\right\rangle},
$$

where $\eta_{f}$ is the $C P$ eigenvalue of the final states. Moreover, for $B_{s}^{0}$ meson decays, a nonzero ratio $(\Delta \Gamma / \Gamma)_{B_{s}^{0}}$ is expected in the SM [61,62]. For $B_{s}^{0} \rightarrow J / \psi \sigma\left(f_{0}\right)$ decays, the third term $A_{\mathrm{CP}}^{\Delta \Gamma_{s}}$ related to the presence of a non-negligible $\Delta \Gamma_{s}$ to describe the $C P$ violation can be defined as follows [62]:

$$
A_{\mathrm{CP}}^{\Delta \Gamma_{s}}=\frac{2 \operatorname{Re}\left(\lambda_{\mathrm{CP}}^{s}\right)}{1+\left|\lambda_{\mathrm{CP}}^{s}\right|^{2}}
$$

The above three quantities describing the $C P$ violations in $B_{s}^{0}$ meson decays shown in Eqs. (60) and (62) satisfy the following relation,

$$
\left|A_{\mathrm{CP}}^{\mathrm{dir}}\right|^{2}+\left|A_{\mathrm{CP}}^{\mathrm{mix}}\right|^{2}+\left|A_{\mathrm{CP}}^{\Delta \Gamma_{s}}\right|^{2}=1 .
$$

The $C P$-violating parameters $\lambda_{\mathrm{CP}}^{d}$ and $\lambda_{\mathrm{CP}}^{s}$ defined for the $B_{d}^{0} \rightarrow J / \psi \sigma\left(f_{0}\right)$ and $B_{s}^{0} \rightarrow J / \psi \sigma\left(f_{0}\right)$ decays can be written explicitly as

$$
\begin{aligned}
& \lambda_{\mathrm{CP}}^{d}=\eta_{f} \frac{V_{t b}^{*} V_{t d}}{V_{t b} V_{t d}^{*}} \cdot \frac{\overline{\mathcal{A}}\left(\bar{B}_{d}^{0} \rightarrow J / \psi \sigma\left(f_{0}\right)\right)}{\mathcal{A}\left(B_{d}^{0} \rightarrow J / \psi \sigma\left(f_{0}\right)\right)}, \\
& \lambda_{\mathrm{CP}}^{s}=\eta_{f} \frac{V_{t b}^{*} V_{t s}}{V_{t b} V_{t s}^{*}} \cdot \frac{\overline{\mathcal{A}}\left(\bar{B}_{s}^{0} \rightarrow J / \psi \sigma\left(f_{0}\right)\right)}{\mathcal{A}\left(B_{s}^{0} \rightarrow J / \psi \sigma\left(f_{0}\right)\right)},
\end{aligned}
$$

with the $C P$ eigenvalue $\eta_{f}=-1$. Based on Eqs. (9)-(12), it is easy to observe that $\lambda_{\mathrm{CP}}^{d}$ and $\lambda_{\mathrm{CP}}^{s}$ are actually determined by the decay amplitudes of $B_{d}^{0} \rightarrow J / \psi f_{q}$ and $B_{s}^{0} \rightarrow J / \psi f_{s}$, respectively. The results of $\lambda_{\mathrm{CP}}^{d}$ and $\lambda_{\mathrm{CP}}^{s}$ can then be read numerically as

$$
\begin{aligned}
& \lambda_{\mathrm{CP}}^{d}=\left(-0.709_{-0.001}^{+0.000}\right)+i\left(0.681_{-0.001}^{+0.000}\right), \\
& \lambda_{\mathrm{CP}}^{s}=\left(-1.000_{-0.000}^{+0.000}\right)-i\left(0.037_{-0.000}^{+0.000}\right) .
\end{aligned}
$$

Therefore, their modules can be read correspondingly as

$$
\begin{aligned}
& \left|\lambda_{\mathrm{CP}}^{d}\right|=0.983_{-0.000}^{+0.001}, \\
& \left|\lambda_{\mathrm{CP}}^{s}\right|=1.001_{-0.000}^{+0.000},
\end{aligned}
$$

which indicate a slightly large (tiny) penguin contamination in these considered $B_{d}^{0}\left(B_{s}^{0}\right)$ decay modes. It is interesting to note that the consistent measurement of $|\lambda|=1.01_{-0.06}^{+0.08} \pm$ 0.03 (the first uncertainty is statical and the second systematic) in the $B_{s}^{0} \rightarrow J / \psi \pi^{+} \pi^{-}$decay was reported very recently by the $\mathrm{LHCb}$ Collaboration [5].

Then, the $C P$ violations of $B_{d, s}^{0} \rightarrow J / \psi \sigma\left(f_{0}\right)$ in the $\mathrm{PQCD}$ approach are as follows,

$$
\begin{aligned}
A_{\mathrm{CP}}^{\mathrm{dir}}\left(B_{d}^{0} \rightarrow J / \psi \sigma\left(f_{0}\right)\right) & \equiv A_{\mathrm{CP}}^{\mathrm{dir}}\left(B_{d}^{0} \rightarrow J / \psi f_{q}\right) \\
& =-1.70_{-0.06}^{+0.06} \times 10^{-2}, \\
A_{\mathrm{CP}}^{\operatorname{mix}}\left(B_{d}^{0} \rightarrow J / \psi \sigma\left(f_{0}\right)\right) & \equiv A_{\mathrm{CP}}^{\mathrm{mix}}\left(B_{d}^{0} \rightarrow J / \psi f_{q}\right) \\
& =0.692_{-0.000}^{+0.001} ; \\
A_{\mathrm{CP}}^{\mathrm{dir}}\left(B_{s}^{0} \rightarrow J / \psi \sigma\left(f_{0}\right)\right) & \equiv A_{\mathrm{CP}}^{\mathrm{dir}}\left(B_{s}^{0} \rightarrow J / \psi f_{s}\right) \\
& =0.733_{-0.044}^{+0.032} \times 10^{-3},
\end{aligned}
$$



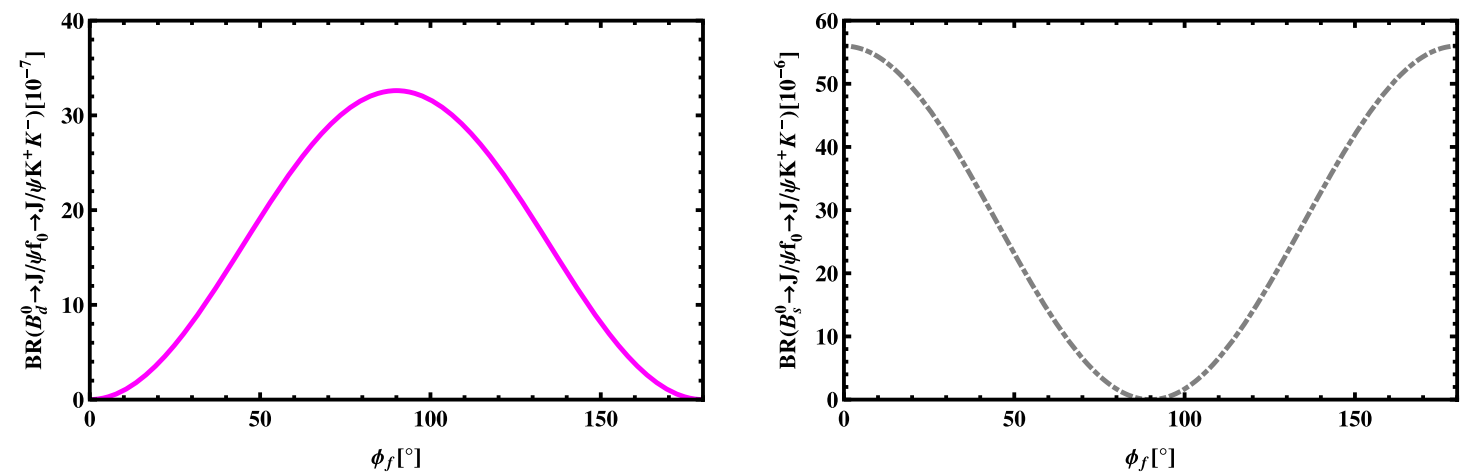

FIG. 3. Dependence on the mixing angle $\phi_{f}$ of the central values for $\operatorname{BR}\left(B_{d, s}^{0} \rightarrow J / \psi f_{0} \rightarrow J / \psi K^{+} K^{-}\right)$in the PQCD approach: The magenta solid (gray dot-dashed) line corresponds to the $B_{d}^{0} \rightarrow J / \psi f_{0}\left(\rightarrow K^{+} K^{-}\right)\left[B_{s}^{0} \rightarrow J / \psi f_{0}\left(\rightarrow K^{+} K^{-}\right)\right]$decay, respectively.

$$
\begin{aligned}
A_{\mathrm{CP}}^{\operatorname{mix}}\left(B_{s}^{0} \rightarrow J / \psi \sigma\left(f_{0}\right)\right) & \equiv A_{\mathrm{CP}}^{\operatorname{mix}}\left(B_{s}^{0} \rightarrow J / \psi f_{s}\right) \\
& =-3.70_{-0.01}^{+0.00} \times 10^{-2}, \\
A_{\mathrm{CP}}^{\Delta \Gamma_{\mathrm{s}}}\left(B_{s}^{0} \rightarrow J / \psi \sigma\left(f_{0}\right)\right) & \equiv A_{\mathrm{CP}}^{\Delta \Gamma_{\mathrm{s}}}\left(B_{s}^{0} \rightarrow J / \psi f_{s}\right) \\
& =-0.999_{-0.000}^{+0.000} .
\end{aligned}
$$

Notice that a $C P$-violating effect $\alpha_{\mathrm{CP}}=\frac{1-\left|\lambda_{f}\right|}{1+\left|\lambda_{f}\right|}$ with $\lambda_{f}$ being the $C P$-violating parameter like $\lambda_{\mathrm{CP}}^{d}$ is fitted as $-58 \pm 46 \times$ $10^{-3}$ for resonance $f_{0}(500)$ in the $B^{0} \rightarrow J / \psi \pi^{+} \pi^{-}$decays [63], which is roughly consistent with our prediction within still large experimental errors.

The above two mixing-induced $C P$ violations, i.e., Eqs. (70) and (72), could be utilized to estimate the penguin impacts on the weak phase $\phi_{d, s}$ in the $B_{d, s}^{0} \rightarrow$ $J / \psi \sigma\left(f_{0}\right)$ decays,

$$
\phi_{d(s)}^{\mathrm{eff}}=-\arg \left[\left(\frac{q}{p}\right)_{d(s)} \frac{\overline{\mathcal{A}}_{f}^{d(s)}}{\mathcal{A}_{f}^{d(s)}}\right]=\phi_{d(s)}^{\mathrm{SM}}+\Delta \phi_{d(s)},
$$

where $\mathcal{A}_{f}^{d(s)}$ and $\overline{\mathcal{A}}_{f}^{d(s)}$ are the decay amplitudes of $B_{d(s)}^{0} \rightarrow$ $J / \psi \sigma\left(f_{0}\right)$ and $\bar{B}_{d(s)}^{0} \rightarrow J / \psi \sigma\left(f_{0}\right)$ decays, respectively. In light of the above-mentioned slightly small or tiny penguin pollution in the $B_{d, s}^{0} \rightarrow J / \psi \sigma\left(f_{0}\right)$ modes, the mixinginduced $C P$-violating asymmetries could be further written approximately as $A_{\mathrm{CP}}^{\mathrm{mix}} \equiv \mathcal{S}_{f} \simeq \sin \phi^{\text {eff }}$, whose evidently nonzero deviations to the $\mathrm{SM}$ one $\sin \phi^{\mathrm{SM}}$ would be helpful to justify the new physics signals beyond SM. It is worth pointing out that only the perturbative expansions at NLO in $\alpha_{s}$ and at leading power in $1 / m_{b}$ are taken into account in the calculations of this work. We extract the quantity $\Delta \phi_{s}$ from our NLO PQCD evaluations with $t$-quark penguin contributions as follows,

$$
\Delta \phi_{s} \approx-0.38_{-0.04}^{+0.06} \times 10^{-3},
$$

where the dominant errors are from the variation of the shape parameter $\omega_{B}$ in the distribution amplitude of $B_{s}^{0}$ meson and the Gegenbauer moments $B_{i}^{s}$ in the distribution amplitude of flavor state $f_{s}$, and various uncertainties have been added in quadrature. The penguin corrections such as $u$-quark and $c$-quark loop contributions are not included here. As discussed in Refs. [51,40], the former correction demands a two-loop calculation for the corresponding amplitude, which is not currently available, while the latter one does not contribute to the quantity $\Delta \phi_{s}$. Therefore, the more precise value about $\Delta \phi_{s}$ extracted from the $B_{s}^{0} \rightarrow$ $J / \psi f_{0}$ mode by including $u$-quark penguin contamination has to be presented elsewhere in the future.

Here, we also calculate the modules of amplitudes for the $B_{d}^{0} \rightarrow J / \psi \sigma, B_{d}^{0} \rightarrow J / \psi f_{0}$, and $B_{s}^{0} \rightarrow J / \psi f_{0}$ decays with definitions as $\left|\mathcal{A}_{d}^{\sigma}\right|,\left|\mathcal{A}_{d}^{f_{0}}\right|$, and $\left|\mathcal{A}_{s}^{f_{0}}\right|$ (in units of $\mathrm{GeV}^{3}$ ),

$$
\begin{aligned}
& \left|\mathcal{A}_{d}^{\sigma}\right| \equiv\left|\mathcal{A}\left(B_{d}^{0} \rightarrow J / \psi \sigma\right)\right|_{\mathrm{PQCD}} \approx 7.03_{-1.03}^{+1.21} \times 10^{-3}, \\
& \left|\mathcal{A}_{d}^{f_{0}}\right| \equiv\left|\mathcal{A}\left(B_{d}^{0} \rightarrow J / \psi f_{0}\right)\right|_{\mathrm{PQCD}} \approx 3.28_{-0.48}^{+0.56} \times 10^{-3}, \\
& \left|\mathcal{A}_{s}^{f_{0}}\right| \equiv\left|\mathcal{A}\left(B_{s}^{0} \rightarrow J / \psi f_{0}\right)\right|_{\mathrm{PQCD}} \approx 2.89_{-0.45}^{+0.56} \times 10^{-2},
\end{aligned}
$$

which result in the ratios $R_{d / s}^{\sigma f_{0}}$ between $\left|\mathcal{A}\left(B_{d}^{0} \rightarrow J / \psi \sigma\right)\right|$ and $\left|\mathcal{A}\left(B_{s}^{0} \rightarrow J / \psi f_{0}\right)\right|$, and $R_{d / s}^{f_{0} f_{0}}$ between $\mid \mathcal{A}\left(B_{d}^{0} \rightarrow\right.$ $\left.J / \psi f_{0}\right) \mid$ and $\left|\mathcal{A}\left(B_{s}^{0} \rightarrow J / \psi f_{0}\right)\right|$ as follows,

$$
\begin{gathered}
R_{d / s}^{\sigma f_{0}} \equiv\left|\frac{\mathcal{A}_{d}^{\sigma}}{\mathcal{A}_{s}^{f_{0}}}\right|_{\mathrm{PQCD}}=0.243_{-0.005}^{+0.003}, \\
R_{d / s}^{f_{0} f_{0}} \equiv\left|\frac{\mathcal{A}_{d}^{f_{0}}}{\mathcal{A}_{s}^{f_{0}}}\right|_{\mathrm{PQCD}}=0.113_{-0.002}^{+0.002} .
\end{gathered}
$$

These two ratios are expected to be helpful to examine the $\mathrm{SU}(3)$ flavor symmetry breaking effects, as well as the useful information on the mixing angle $\phi_{f}$, in these considered $B_{d}^{0} \rightarrow J / \psi \sigma\left(f_{0}\right)$ and $B_{s}^{0} \rightarrow J / \psi f_{0}$ decays.

Last but not least, it is noted that the scalar meson $f_{0}$ decays largely into $\pi^{+} \pi^{-}$but can also decay into $K^{+} K^{-}$. Therefore, some useful information about this $f_{0}$ meson 
could also be hinted from the analysis of $B_{d, s}^{0} \rightarrow J / \psi f_{0} \rightarrow$ $J / \psi K^{+} K^{-}$decays. The dependence of $\operatorname{BR}\left(B_{d, s}^{0} \rightarrow\right.$ $\left.J / \psi f_{0}\left(\rightarrow K^{+} K^{-}\right)\right)$on the mixing angle $\phi_{f}$ is plotted in Fig. 3. According to $\mathcal{B}\left(f_{0} \rightarrow K^{+} K^{-}\right)=0.16_{-0.05}^{+0.04}$, the branching ratios of $B_{d, s}^{0} \rightarrow J / \psi f_{0}\left(\rightarrow K^{+} K^{-}\right)$, as a byproduct, could be easily obtained at $\phi_{f} \approx 25^{\circ}$ as follows,

$$
\left.\mathrm{BR}\left(B_{d}^{0} \rightarrow J / \psi f_{0}, f_{0} \rightarrow K^{+} K^{-}\right)\right|_{\mathrm{PQCD}}=0.58_{-0.29}^{+0.31} \times 10^{-6},
$$

$\left.\mathrm{BR}\left(B_{s}^{0} \rightarrow J / \psi f_{0}, f_{0} \rightarrow K^{+} K^{-}\right)\right|_{\mathrm{PQCD}}=0.46_{-0.23}^{+0.26} \times 10^{-4}$.

Then, the interesting ratios could be further derived as

$$
\begin{aligned}
R_{f_{0} / \rho}^{\mathrm{Th}}(K) & \left.\equiv \frac{\mathrm{BR}\left(B_{d}^{0} \rightarrow J / \psi f_{0}\right) \mathcal{B}\left(f_{0} \rightarrow K^{+} K^{-}\right)}{\operatorname{BR}\left(B_{d}^{0} \rightarrow J / \psi \rho^{0}\right) \mathcal{B}\left(\rho^{0} \rightarrow \pi^{+} \pi^{-}\right)}\right|_{\mathrm{PQCD}} \\
& =0.021_{-0.006}^{+0.003},
\end{aligned}
$$

$$
\begin{aligned}
R_{f_{0} / \phi}^{\mathrm{Th}}(K) & \left.\equiv \frac{\mathrm{BR}\left(B_{s}^{0} \rightarrow J / \psi f_{0}\right) \mathcal{B}\left(f_{0} \rightarrow K^{+} K^{-}\right)}{\operatorname{BR}\left(B_{s}^{0} \rightarrow J / \psi \phi\right) \mathcal{B}\left(\phi \rightarrow K^{+} K^{-}\right)}\right|_{\mathrm{PQCD}} \\
& =0.092_{-0.026}^{+0.014},
\end{aligned}
$$

which are expected to be tested in the measurements at LHCb and/or Belle-II experiments. Furthermore, the relevant examinations provide more supplementary constraints on the mixing angle $\phi_{f}$. By the way, frankly speaking, the $B_{s}^{0} \rightarrow J / \psi f_{0}\left(\rightarrow K^{+} K^{-}\right)$branching ratio measurement is still necessary, although it is very difficult experimentally as $f_{0}$ is buried under the tail of $\phi$ (see Fig. 7 in Ref. [64] for example) [65].

Finally, two more comments are as follows:

(a) For final state interactions: As mentioned in the above, we just include the short distance contributions that can be perturbatively calculated in this work. Other possible contributions such as rescattering effects or final state interactions are not considered yet, though they are generally believed to affect the predictions of the observables potentially.

(b) For possible tetraquark structure: In principle, we also need to make some calculations to help identify the possible tetraquark structure of $\sigma$ and $f_{0}$. However, the essential inputs such as light-cone distribution amplitudes are still unavailable now. Therefore, we cannot obtain the information about the possible tetraquark components straightforwardly from the perturbative evaluations in the heavy $B$ meson decays currently.

The above two issues have to be left for future investigations after precise measurements experimentally and related improvements theoretically.

\section{SUMMARY}

As an ideally alternative channel with no need of angular decomposition, the $B_{s}^{0} \rightarrow J / \psi f_{0}$ decay is expected to have great potential to reduce errors in the extraction of the $B_{s}^{0}-\bar{B}_{s}^{0}$ mixing phase $\phi_{s}$, which will help us to search for the new physics beyond SM associated with the precision measurements performed at the upgraded $\mathrm{LHCb}$ and/or the ongoing Belle-II experiments. The quantitative exploration demands the reliable calculations about the corresponding decay amplitude. As a possible reference, we made the investigations by assuming $f_{0}$ as the ground scalar meson in the two-quark picture, where it is believed that $\sigma$ and $f_{0}$ could mix with each other in the quark-flavor basis with a single mixing angle $\phi_{f}$. Up to now, $\phi_{f}$ has not been determined definitely, although several studies at both theoretical and experimental aspects have been presented.

Motivated by the global agreement on the observables of the $B \rightarrow J / \psi V$ decays between the data and the PQCD approach at NLO accuracy, we extended that formalism to the $B_{d, s}^{0} \rightarrow J / \psi \sigma\left(f_{0}\right)$ channels. The NLO PQCD predictions on the $C P$-averaged branching ratios for the $B_{d, s}^{0} \rightarrow$ $J / \psi \sigma / f_{0}\left(\rightarrow \pi^{+} \pi^{-}\right)$decays and the relative ratios generally agree with the current data or upper limits within still large theoretical errors around the mixing angle $\phi_{f} \approx 25^{\circ}$ with a twofold ambiguity. It is stressed that this twofold ambiguity could be resolved in the $B \rightarrow M \sigma\left(f_{0}\right)$ decays with $M$ being certain light or open-charmed hadrons due to the constructive or destructive interferences between $B \rightarrow M f_{q}$ and $B \rightarrow M f_{s}$ decays. Several interesting observables such as branching ratios, relative ratios, and $C P$-violating asymmetries for the $B_{d, s}^{0} \rightarrow J / \psi \sigma\left(f_{0}\right)$ decays are then predicted in the PQCD approach at NLO level. They could be utilized to either constrain the mixing angle $\phi_{f}$ or estimate the SU(3) flavor symmetry breaking effects. As a byproduct, the branching ratios of $B_{d, s}^{0} \rightarrow J / \psi f_{0}(\rightarrow$ $K^{+} K^{-}$) are also predicted in this work. These given predictions about the $B_{d, s}^{0} \rightarrow J / \psi \sigma\left(f_{0}\right)$ decays await the future examinations with high precision.

\section{ACKNOWLEDGMENTS}

X.L. thanks Professor Hai-Yang Cheng and Professor Hsiang-nan Li for helpful discussions. The authors are very grateful to Professor Sheldon Stone for his enlightening discussions and valuable comments on the manuscript. This work is supported in part by the National Natural Science Foundation of China under Grants No. 11575151, No. 11775117, No. 11875033, No. 11705159, No. 11447032, and No. 11765012, by the Qing Lan Project of Jiangsu Province (Grant No. 9212218405), by the Natural Science Foundation of Shandong Province (Grants No. ZR2016JL001, No. ZR2018JL001, and No. ZR2019JQ04), and by the Research Fund of Jiangsu Normal University (Grant No. HB2016004). 


\section{APPENDIX: EFFECTIVE WILSON COEFFICIENTS}

As was pointed out in Ref. [50], for these considered $B_{d, s}^{0} \rightarrow J / \psi \sigma\left(f_{0}\right)$ decays, only the vertex corrections contribute at the currently known NLO level, in which their effects can be absorbed into the Wilson coefficients associated with the factorizable emission contributions $[66,67]$,

$$
\begin{gathered}
\tilde{a}_{2}=C_{1}+\frac{C_{2}}{N_{c}}+\frac{\alpha_{s}}{4 \pi} \frac{C_{F}}{N_{c}} C_{2}\left(-18+12 \ln \frac{m_{b}}{\mu}+f_{I}^{0}\right), \\
\tilde{a}_{3}=C_{3}+\frac{C_{4}}{N_{c}}+\frac{\alpha_{s}}{4 \pi} \frac{C_{F}}{N_{c}} C_{4}\left(-18+12 \ln \frac{m_{b}}{\mu}+f_{I}^{0}\right), \\
\tilde{a}_{5}=C_{5}+\frac{C_{6}}{N_{c}}+\frac{\alpha_{s}}{4 \pi} \frac{C_{F}}{N_{c}} C_{6}\left(6-12 \ln \frac{m_{b}}{\mu}-f_{I}^{0}\right),
\end{gathered}
$$

$$
\tilde{a}_{7}=C_{7}+\frac{C_{8}}{N_{c}}+\frac{\alpha_{s}}{4 \pi} \frac{C_{F}}{N_{c}} C_{8}\left(6-12 \ln \frac{m_{b}}{\mu}-f_{I}^{0}\right),
$$

$$
\tilde{a}_{9}=C_{9}+\frac{C_{10}}{N_{c}}+\frac{\alpha_{s}}{4 \pi} \frac{C_{F}}{N_{c}} C_{10}\left(-18+12 \ln \frac{m_{b}}{\mu}+f_{I}^{0}\right),
$$

with the function $f_{I}^{0}$,

$$
f_{I}^{0}=f_{I}+g_{I}(1-z)
$$

where $z \equiv r_{d, s}^{2}=m_{J / \psi}^{2} / m_{B_{d, s}^{0}}^{2}$ and the functions $f_{I}$ and $g_{I}$ read as [67]

$$
\begin{aligned}
f_{I}= & \frac{2 \sqrt{2 N_{c}}}{f_{J / \psi}} \int_{0}^{1} d x_{2} \phi_{J / \psi}^{L}\left(x_{2}\right)\left\{\frac{2 z x_{2}}{1-z\left(1-x_{2}\right)}+\left(3-2 x_{2}-8 x_{2}^{2}\right) \frac{\ln x_{2}}{1-x_{2}}\right. \\
& +\left(-\frac{3}{1-z x_{2}}+\frac{1+8 x_{2}}{1-z\left(1-x_{2}\right)}-\frac{2 z x_{2}}{\left[1-z\left(1-x_{2}\right)\right]^{2}}\right) z x_{2} \ln z x_{2} \\
& \left.+\left(3(1-z)+2 z x_{2}-8 z x_{2}^{2}+\frac{2 z^{2} x_{2}^{2}}{1-z\left(1-x_{2}\right)}\right) \frac{\ln (1-z)-i \pi}{1-z\left(1-x_{2}\right)}\right\},
\end{aligned}
$$

and

$$
\begin{aligned}
g_{I}= & \frac{2 \sqrt{2 N_{c}}}{f_{J / \psi}} \int_{0}^{1} d x_{2} \phi_{J / \psi}^{L}\left(x_{2}\right)\left\{\frac{4 x_{2}\left(2 x_{2}-1\right)}{(1-z)\left(1-x_{2}\right)} \ln x_{2}+\frac{z x_{2}}{\left[1-z\left(1-x_{2}\right)\right]^{2}} \ln (1-z)\right. \\
& +\left(\frac{1}{\left(1-z x_{2}\right)^{2}}-\frac{1}{\left[1-z\left(1-x_{2}\right)\right]^{2}}-\frac{8 x_{2}}{(1-z)\left(1-z x_{2}\right)}\right. \\
& \left.\left.+\frac{2\left(1+z-2 z x_{2}\right)}{(1-z)\left(1-z x_{2}\right)^{2}}\right) z x_{2} \ln z x_{2}-i \pi \frac{z x_{2}}{\left[1-z\left(1-x_{2}\right)\right]^{2}}\right\},
\end{aligned}
$$

respectively.

[1] S. Stone and L. Zhang, Phys. Rev. D 79, 074024 (2009).

[2] S. Stone and L. Zhang, arXiv:0909.5442.

[3] S. Stone, Proc. Sci. FPCP2010 (2010) 011.

[4] M. Tanabashi et al. (Particle Data Group), Phys. Rev. D 98, 030001 (2018); C. Amsler, S. Eidelman, T. Gutsche, C. Hanhart, S. Spanier, and N. A. Törnqvist, Rev. Part. Phys. 1, 658 (2018).

[5] R. Aaij et al. (LHCb Collaboration), arXiv:1903.05530.

[6] R. Aaij et al. (LHCb Collaboration), Phys. Rev. D 87, 052001 (2013).
[7] R. Aaij et al. (LHCb Collaboration), Phys. Rev. D 90, 012003 (2014).

[8] J. Li et al. (Belle Collaboration), Phys. Rev. Lett. 106, 121802 (2011).

[9] T. Aaltonen et al. (CDF Collaboration), Phys. Rev. D 84, 052012 (2011).

[10] R. Aaij et al. (LHCb Collaboration), Phys. Rev. D 89, 092006 (2014).

[11] K. De Bruyn, R. Fleischer, R. Knegjens, P. Koppenburg, M. Merk, and N. Tuning, Phys. Rev. D 86, 014027 (2012). 
[12] R. Aaij et al. (LHCb Collaboration), Phys. Lett. B 698, 115 (2011).

[13] V. M. Abazov et al. (D0 Collaboration), Phys. Rev. D 85, 011103 (2012).

[14] V. Khachatryan et al. (CMS Collaboration), Phys. Lett. B 756, 84 (2016).

[15] Y. Amhis et al. (HFLAV Collaboration), Eur. Phys. J. C 77, 895 (2017).

[16] R. Aaij et al. (LHCb Collaboration), Phys. Rev. D 86, 052006 (2012).

[17] W. Wang, Phys. Lett. B 759, 501 (2016).

[18] S. Godfrey and J. Napolitano, Rev. Mod. Phys. 71, 1411 (1999).

[19] F. E. Close and N. A. Tornqvist, J. Phys. G 28, R249 (2002).

[20] C. Amsler and N. A. Tornqvist, Phys. Rep. 389, 61 (2004).

[21] E. Klempt and A. Zaitsev, Phys. Rep. 454, 1 (2007).

[22] V. Crede and C. A. Meyer, Prog. Part. Nucl. Phys. 63, 74 (2009).

[23] W. Ochs, J. Phys. G 40, 043001 (2013).

[24] S. Stone and L. Zhang, Phys. Rev. Lett. 111, 062001 (2013).

[25] P. Colangelo, F. De Fazio, and W. Wang, Phys. Rev. D 81, 074001 (2010).

[26] P. Colangelo, F. De Fazio, and W. Wang, Phys. Rev. D 83, 094027 (2011).

[27] O. Leitner, J.-P. Dedonder, B. Loiseau, and B. El-Bennich, Phys. Rev. D 82, 076006 (2010).

[28] R. Fleischer, R. Knegjens, and G. Ricciardi, Eur. Phys. J. C 71, 1832 (2011).

[29] J. W. Li, D. S. Du, and C. D. Lü, Eur. Phys. J. C 72, 2229 (2012).

[30] W. H. Liang and E. Oset, Phys. Lett. B 737, 70 (2014).

[31] M. Bayar, W. H. Liang, and E. Oset, Phys. Rev. D 90, 114004 (2014).

[32] W. F. Wang, H. n. Li, W. Wang, and C. D. Lü, Phys. Rev. D 91, 094024 (2015).

[33] F. E. Close and A. Kirk, Phys. Rev. D 91, 114015 (2015).

[34] J. T. Daub, C. Hanhart, and B. Kubis, J. High Energy Phys. 02 (2016) 009.

[35] S. Ropertz, C. Hanhart, and B. Kubis, Eur. Phys. J. C 78, 1000 (2018).

[36] Y.-Y. Keum, H.-n. Li, and A. I. Sanda, Phys. Lett. B 504, 6 (2001).

[37] Y. Y. Keum, H.-N. Li, and A. I. Sanda, Phys. Rev. D 63, 054008 (2001).

[38] C.-D. Lu, K. Ukai, and M.-Z. Yang, Phys. Rev. D 63, 074009 (2001).
[39] C.-D. Lu and M.-Z. Yang, Eur. Phys. J. C 23, 275 (2002).

[40] X. Liu, W. Wang, and Y. Xie, Phys. Rev. D 89, 094010 (2014).

[41] J. Botts and G. F. Sterman, Nucl. Phys. B325, 62 (1989).

[42] H. n. Li and G. F. Sterman, Nucl. Phys. B381, 129 (1992).

[43] H. n. Li, Phys. Rev. D 66, 094010 (2002).

[44] H. n. Li and K. Ukai, Phys. Lett. B 555, 197 (2003).

[45] X. Liu, H.-n. Li, and Z. J. Xiao, Phys. Rev. D 86, 011501 (2012).

[46] H. Y. Cheng, Phys. Rev. D 67, 034024 (2003).

[47] H. Y. Cheng, C. K. Chua, and K. C. Yang, Phys. Rev. D 73, 014017 (2006).

[48] H. Y. Cheng, C. K. Chua, K. C. Yang, and Z. Q. Zhang, Phys. Rev. D 87, 114001 (2013).

[49] C.-H. Chen and H.-N. Li, Phys. Rev. D 71, 114008 (2005).

[50] X. Liu, Z.-Q. Zhang, and Z. J. Xiao, Chin. Phys. C 34, 937 (2010).

[51] H.-n. Li and S. Mishima, J. High Energy Phys. 03 (2007) 009.

[52] X. Liu and Z. J. Xiao, Phys. Rev. D 89, 097503 (2014).

[53] H. Y. Cheng and K. C. Yang, Phys. Rev. D 71, 054020 (2005).

[54] H. n. Li and S. Mishima, Phys. Rev. D 74, 094020 (2006).

[55] G. Buchalla, A. J. Buras, and M. E. Lautenbacher, Rev. Mod. Phys. 68, 1125 (1996).

[56] B. Aubert et al. (BABAR Collaboration), Phys. Rev. D 74, 032003 (2006).

[57] M. Ablikim et al. (BES Collaboration), Phys. Rev. D 70, 092002 (2004).

[58] M. Ablikim et al. (BES Collaboration), Phys. Rev. D 72, 092002 (2005).

[59] K. M. Ecklund et al. (CLEO Collaboration), Phys. Rev. D 80, 052009 (2009).

[60] H. Y. Cheng, Phys. Rev. D 68, 014015 (2003).

[61] M. Beneke, G. Buchalla, C. Greub, A. Lenz, and U. Nierste, Phys. Lett. B 459, 631 (1999).

[62] L. Fernandez, Ecole Polytechnique, Report No. CERNTHESIS-2006-042.

[63] R. Aaij et al. (LHCb Collaboration), Phys. Lett. B 742, 38 (2015).

[64] R. Aaij et al. (LHCb Collaboration), J. High Energy Phys. 08 (2017) 037.

[65] S. Stone (private communication).

[66] J. Chay and C. Kim, arXiv:hep-ph/0009244.

[67] H. Y. Cheng and K. C. Yang, Phys. Rev. D 63, 074011 (2001). 